\title{
The branch locus for one-dimensional Pisot tiling spaces
}

\author{
by \\ Marcy Barge (Bozeman, MT), Beverly Diamond (Charleston, SC) \\ and Richard Swanson (Bozeman, MT)
}

\begin{abstract}
If $\varphi$ is a Pisot substitution of degree $d$, then the inflation and substitution homeomorphism $\Phi$ on the tiling space $\mathcal{T}_{\Phi}$ factors via geometric realization onto a $d$-dimensional solenoid. Under this realization, the collection of $\Phi$-periodic asymptotic tilings corresponds to a finite set that projects onto the branch locus in a $d$-torus. We prove that if two such tiling spaces are homeomorphic, then the resulting branch loci are the same up to the action of certain affine maps on the torus.
\end{abstract}

1. Introduction. In this paper we introduce the branch locus, a new topological invariant for one-dimensional Pisot substitution tiling spaces. A substitution on $n$ letters is a map from an alphabet $\mathcal{A}=\{1, \ldots, n\}$ into $\mathcal{A}^{*}$, where $\mathcal{A}^{*}$ is the collection of finite and nonempty words from $\mathcal{A}$. The abelianization matrix of $\varphi$ is defined as $A=\left(a_{i j}\right)$, where $a_{i j}=$ number of occurrences of $i$ in $\varphi(j)$. The substitution $\varphi$ is Pisot provided the PerronFrobenius eigenvalue $\lambda$ of $A$ is a Pisot-Vijayaraghavan number $(\lambda>1$ and all algebraic conjugates of $\lambda$ are strictly inside the unit circle). The degree of $\varphi$ is the degree of the minimal polynomial of $\lambda$.

Associated with any substitution $\varphi$, there is a tiling space $\mathcal{T}_{\varphi}$ consisting of certain tilings of the real line; if $\left(\lambda_{1}, \ldots, \lambda_{n}\right)$ is a positive left eigenvector of $A$, then $\mathcal{T}_{\varphi}$ consists of all tilings of $\mathbb{R}$ by translates of the prototiles $P_{i}=\left[0, \lambda_{i}\right], i=1, \ldots, n$, with the property that the word spelled out by any finite patch of consecutive tiles in the tiling occurs as a factor of $\varphi^{m}(i)$ for some $i \in \mathcal{A}$ and $m \in \mathbb{N}$. The substitution $\varphi$ is primitive if for some $m \in \mathbb{N}$, every entry of $A^{m}$ is strictly positive, and aperiodic if no element of $\mathcal{T}_{\varphi}$ is periodic under translation. For the remainder of the paper, all substitutions will be assumed primitive and aperiodic.

2000 Mathematics Subject Classification: Primary 37B05; Secondary 37A30, 37B50, $54 \mathrm{H} 20$.

Key words and phrases: tiling space, Pisot substitution, geometric realization, branch locus. 
Define the topology of $\mathcal{T}_{\varphi}$ by stipulating that two tilings are close provided a small translate of one is identical to the other in a large neighborhood of the origin. Under the assumption that $\varphi$ is primitive and aperiodic, $\mathcal{T}_{\varphi}$ is a continuum (a compact, connected metric space). For such a $\varphi$, inflation and substitution is the homeomorphism $\Phi: \mathcal{T}_{\varphi} \rightarrow \mathcal{T}_{\varphi}$ that replaces each tile $t+P_{i}=\left[t, \lambda_{i}+t\right]$ of a tiling $T$ in $\mathcal{T}_{\varphi}$ by the patch $\left[\lambda t, \lambda \lambda_{i}+\lambda t\right]$ tiled by translates of prototiles following the pattern of the word $\varphi(i)$. There is also a minimal and uniquely ergodic $\mathbb{R}$-action on $\mathcal{T}_{\varphi}$, called the translation flow, given by $T=\left\{T_{i}\right\}_{i=-\infty}^{\infty} \mapsto T-t:=\left\{T_{i}-t\right\}_{i=-\infty}^{\infty}$ for $t \in \mathbb{R}$.

The topology of a substitution tiling space is of interest for a number of reasons. Physics provides one source of motivation. Suppose that $T$ is a tiling in the substitution tiling space $\mathcal{T}_{\varphi}$. Placing an atom at the end of each tile creates a one-dimensional material which is called a quasi-crystal if its diffraction spectrum is pure point (the atoms must be "weighted" according to the tile types they lie in). Bombieri and Taylor ([BT]) proved that if $\varphi$ is Pisot, such a material has a nontrivial discrete component in its spectrum. Whether such a material has pure point spectrum when $\varphi$ is irreducible unimodular Pisot (i.e., $\varphi$ is Pisot with degree $(\lambda)=d=n=|\mathcal{A}|$, and $\operatorname{det}(A)= \pm 1$ ) remains an open question. Lee, Moody and Solomyak ([LMS]) have proved that the diffraction spectrum of the material is pure point if and only if the dynamical spectrum of the translation flow on $\mathcal{T}_{\varphi}$ is pure discrete. It follows from [BSw2] that if $\mathcal{T}_{\varphi}$ and $\mathcal{T}_{\psi}$ are homeomorphic tiling spaces, then the tiling flow on $\mathcal{T}_{\varphi}$ is pure discrete if and only if the tiling flow on $\mathcal{T}_{\psi}$ is pure discrete. That is, the question of whether or not a one-dimensional material built from a substitution has pure point diffraction spectrum is a topological question about the corresponding tiling space.

Substitution tiling spaces also arise in the study of hyperbolic attractors. R. F. Williams ([Wi]) proved that every hyperbolic one-dimensional attractor is topologically conjugate with the shift map on the inverse limit of an expanding endomorphism of a branched one-manifold, and, with minor restrictions on the map of the branched one-manifold, all such inverse limits can be realized as hyperbolic attractors. More recently, Anderson and Putnam ([AP]) proved that inflation and substitution on a one-dimensional substitution tiling space is conjugate with the shift on the inverse limit of an expanding endomorphism of a branched one-manifold. As a consequence, every orientable hyperbolic one-dimensional attractor is either a substitution tiling space, for which the underlying manifold is branched, or a classical solenoid, for which the underlying manifold is the circle. Modeling an attractor as a tiling space provides a much clearer view of its global topology than one gets from considering an inverse limit description: moving along an arc component in the attractor is simply translating a tiling, and the patterns of consecutive tiles determine the recurrence properties of the translates. 
Although the "inverse limit on branched manifolds" description of tiling spaces will not play an explicit role in this paper, the intuitive content of our main result, and the rationale for the terminology "branch locus" that we introduce, has its origin in that description. There are, of course, no actual branch points in a tiling space: in the one-dimensional case, every point has a neighborhood that is homeomorphic with the product of an arc and a Cantor set. Nevertheless, an inverse limit description of the tiling space gives a sequence of approximating branched one-manifolds. In the limit, the ghost of the branches can be observed in the existence of asymptotic composants: two distinct tilings $T, T^{\prime}$ are asymptotic provided $d\left(T-t, T^{\prime}-t\right) \rightarrow 0$ as $t \rightarrow \infty$ or as $t \rightarrow-\infty$. The arc components of asymptotic tilings are called asymptotic composants. Partially sewing up such asymptotic composants results in a space that does have branching: the new, branched, space corresponds to an inverse limit on a branched manifold with periodic branch points.

Although this "ghost branching" in $\mathcal{T}_{\varphi}$ seems to have no clear location, we will see that, in case the substitution is Pisot, the branching occurs in well defined relative geometrical patterns. The appropriate underlying geometry is that of the $d$-dimensional torus, where $d$ is the degree of $\lambda$. Our main result is that if $\mathcal{T}_{\varphi}$ and $\mathcal{T}_{\psi}$ are homeomorphic tiling spaces, then their branch loci, nonempty finite sets of points in the $d$-torus that we define in $\S 2$, are equal modulo the action of a certain collection of affine endomorphisms of the torus. Thus the branch locus becomes a topological invariant. We illustrate this by distinguishing pairs of tiling spaces that are otherwise difficult to tell apart (see $\S 3$ ).

The idea for considering "branching" in one-dimensional tiling spaces arose in discussions the first author had with Søren Eilers regarding the topological content of the $K_{0}$-group of the Matsumoto algebra associated with a substitution. Eilers, Restorff and Ruiz ([ERR]) have shown that this (ordered) group is also a complete invariant of the Matsumoto algebra and, consequently, the Matsumoto algebra is a topological invariant of the tiling space.

How is the Matsumoto algebra (equivalently, its $K_{0}$-group) reflected in the topology of the tiling space? In $\S 5$ we show that the branch locus provides a partial answer. We use the branch locus to define the "Pisot part of the augmented dimension group", an ordered group that is a flow equivalence invariant of the substitution and an ordered subgroup of the augmented cohomology group of the tiling space, which, in turn, is closely related to the Matsumoto $K_{0}$-group (see [CE1], [CE2] for a description of the Matsumoto $K_{0}$-group in the substitutive setting and [BSm] for an account of the relationship between the Matsumoto $K_{0}$-group of a substitutive system and the augmented cohomology of the associated tiling space). 
2. Geometric realization. For convenience, we will use the "strand space" model for the tiling space (see $[\mathrm{BK}]$ and $[\mathrm{BBK}]$ ) which we recall now. Let $\varphi$ be a Pisot substitution of degree $d$ on $n$ letters with abelianization $A$ and Perron-Frobenius eigenvalue $\lambda$. There is a (unique) $A$-invariant decomposition $\mathbb{R}^{n}=V \oplus W$ such that $V$ contains a right Perron-Frobenius eigenvector $\omega$ associated with $\lambda$ and $\operatorname{dim} V=d\left(^{1}\right)$. We can always choose rational bases for $V$ and $W$. There is a further $\left.A\right|_{V^{-}}$-invariant splitting $V=\mathbb{E}^{u} \oplus \mathbb{E}^{s}$ obtained by letting $\mathbb{E}^{s}$ be the space orthogonal to a left Perron-Frobenius eigenvector of $\left.A\right|_{V}$ and $\mathbb{E}^{u}$ be the span of $\omega$. Let $\operatorname{pr}_{V}: \mathbb{R}^{n} \rightarrow V, \operatorname{pr}_{s}: V \rightarrow \mathbb{E}^{s}$, and $\operatorname{pr}_{u}: V \rightarrow \mathbb{E}^{u}$ denote the projections, resp., along $W, \mathbb{E}^{u}$ and $\mathbb{E}^{s}$, and let $\Gamma$ denote the $A$-invariant lattice $\operatorname{pr}_{V} \mathbb{Z}^{n}$.

If $e_{i}, i=1, \ldots, n$, are the standard basis vectors in $\mathbb{R}^{n}$, define $v_{i}:=$ $\operatorname{pr}_{V}\left(e_{i}\right)$, and let $\sigma_{i}:=\left\{t v_{i}: 0 \leq t \leq 1\right\}$ denote the oriented segment representing $v_{i}$. Even if $\sigma_{i}=\sigma_{j}$ for some $i \neq j$, we wish to distinguish between these segments: we call $\sigma_{i}$ a (labeled) segment of type $i$. An oriented broken line $\gamma=\left\{\sigma_{i_{k}}+x_{k}\right\}, x_{k} \in V$, consisting of a collection of translated copies of the basic segments meeting tip-to-tail and with connected union, will be called a strand. We denote the space of bi-infinite strands in $V$ by

$$
\mathcal{F}:=\{\gamma: \gamma \text { is a bi-infinite strand in } V\} .
$$

The substitution $\varphi$ induces the inflation and substitution map $\Phi: \mathcal{F} \rightarrow \mathcal{F}$ as follows: for each edge (translated segment) $\sigma_{i_{k}}+x_{k}$ in strand $\gamma$, replace that edge by the collection of edges

$$
\sigma_{j_{1}}+A x_{k}, \quad \sigma_{j_{2}}+A x_{k}+v_{j_{1}}, \ldots, \sigma_{j_{l}}+A x_{k}+v_{j_{1}}+\cdots+v_{j_{l-1}},
$$

where $\varphi\left(i_{k}\right)=j_{1} j_{2} \cdots j_{l}$. That is, $\Phi(\gamma)$ is obtained by applying the linear map $A$ to each edge of $\gamma$, then breaking up the image into translated segments following the pattern determined by applying $\varphi$ to the type of the edge.

For $R>0$, let $\mathcal{F}^{R}$ denote the subset of $\mathcal{F}$ consisting of those strands all of whose edges are within distance $R$ of $\mathbb{E}^{u}$. There is then an $R_{0}$ such that

$$
\mathcal{F}_{\varphi}:=\bigcap_{n \geq 0} \Phi^{n}\left(\mathcal{F}^{R}\right)
$$

is independent of $R \geq R_{0}$. The set $\mathcal{F}_{\varphi}$ has a natural metric topology in which $\gamma$ and $\gamma^{\prime}$ are close if a small translate of $\gamma$ by a vector in $V$ lines up exactly with $\gamma^{\prime}$, segment types being considered, in a large neighborhood of the origin. With respect to this topology, $\Phi$ and the translation flow,

$$
\gamma=\left\{\sigma_{i_{k}}+x_{k}\right\} \mapsto \gamma-t:=\left\{\sigma_{i_{k}}+x_{k}-t \omega\right\},
$$

are continuous.

$\left(^{1}\right) V$ is the kernel of $m(A)$, where $m(x)$ denotes the minimal polynomial of $\lambda$. 
In some cases there may be a few translation orbits in $\mathcal{F}_{\varphi}$ that correspond to strands with acceptable heads and tails but that are joined in an unnatural way (this happens, for instance, when there are letters $a$ and $b$ with $\varphi(a)=a \cdots, \varphi(b)=\cdots b$, but the word $b a$ never occurs in $\varphi^{m}(i), i \in \mathcal{A}$, $m \in \mathbb{N})$. We eliminate these chimeras by defining $\mathcal{T}_{\varphi}$ to be the $\omega$-limit set, under translation flow, of any $\gamma \in \mathcal{F}_{\varphi}$. That is, for any $\gamma \in \mathcal{F}_{\varphi}$,

$$
\mathcal{T}_{\varphi}:=\bigcap_{T>0} \operatorname{cl}\{\gamma-t: t \in[T, \infty)\} .
$$

Inflation and substitution, $\Phi: \mathcal{T}_{\varphi} \rightarrow \mathcal{T}_{\varphi}$, is a homeomorphism from $\mathcal{T}_{\varphi}$ onto itself, and the translation flow on $\mathcal{T}_{\varphi}$ is minimal and uniquely ergodic.

One reason for using the rather elaborate strand space model of the tiling space is that it permits a simple and concrete definition of geometric realization. One could factor the tiling dynamics onto those of a solenoid, by choosing the lattice $\Gamma$ in $V$ and mapping $\gamma \in \mathcal{T}_{\varphi}$ to

$$
\left(\min I_{0}+\Gamma, \min I_{1}+\Gamma, \ldots\right) \in \lim _{\longleftarrow} F_{\left.A\right|_{V}},
$$

where $F_{\left.A\right|_{V}}: V / \Gamma \rightarrow V / \Gamma$ is defined by $F_{\left.A\right|_{V}}(v+\Gamma)=A v+\Gamma, I_{k}$ is any edge in $\Phi^{-k}(\gamma)$ and $\min I_{k}$ is its initial vertex. This does give a well defined surjection of $\mathcal{T}_{\varphi}$ onto the $d$-dimensional solenoid $\lim _{F_{\left.A\right|_{V}}}$ that semiconjugates inflation and substitution with the shift, as well as translation flow with a Kronecker action. However, to maximize the size of the factor, we will define a coarser, more natural lattice. Toward this end, denote the collection of return vectors by

$$
\begin{aligned}
\Theta(i):=\left\{v \in \Gamma: \text { there exists } \gamma \in \mathcal{T}_{\varphi} \text { containing edges } I, I^{\prime},\right. \\
\\
\text { each of type } \left.i \text {, with } I^{\prime}=I+v\right\} .
\end{aligned}
$$

It is not difficult to show that the subgroup

$$
\Sigma_{\infty}:=\left\langle\bigcup_{k \in \mathbb{Z}}\left(\left.A\right|_{V}\right)^{k} \Theta(i)\right\rangle
$$

of $V$ generated by $\bigcup_{k \in \mathbb{Z}}\left(\left.A\right|_{V}\right)^{k} \Theta(i)$ is independent of $i \in \mathcal{A}$. The return lattice

$$
\Sigma:=\Sigma_{\infty} \cap \Gamma
$$

is invariant under $A$ and also of rank $d$.

Note: If $A$ is irreducible $(d=n)$, then $\Sigma=\Gamma=\mathbb{Z}^{n}$. In $\S 3$, we will see an example where $\Sigma$ is strictly coarser than $\Gamma$.

To define geometric realization onto a solenoid determined by $\Sigma$, we must determine some appropriate translations. We begin by noticing that for each $i, j \in \mathcal{A}$, there is a well defined "transition vector" $w_{i j} \in \Gamma / \Sigma$ such that if $\gamma \in \mathcal{T}_{\varphi}$ and $I$ and $J$ are edges of $\gamma$ of types $i$ and $j$ resp., then

$$
(\min J-\min I) \bmod \Sigma=w_{i j} \text {. }
$$


Then, since $w_{i j}+w_{j k}+w_{k i}=0$ for all $i, j, k \in \mathcal{A}$, there are $u_{i} \in \Gamma / \Sigma$, for $i \in \mathcal{A}$, such that for all $i, j$,

$$
w_{i j}=u_{j}-u_{i} .
$$

We may then normalize the $u_{i}$ so that

$$
A u_{i}=u_{i^{\prime}}
$$

for all $i \in \mathcal{A}$, where $i^{\prime}$ denotes the initial letter of $\varphi(i)$. Letting $F_{\left.A\right|_{V}}$ denote the map induced by $A$ on the torus $V / \Sigma$, we have a well defined map $g_{\varphi}$ : $\mathcal{T}_{\varphi} \rightarrow \underset{\lim }{\longleftarrow} F_{\left.A\right|_{V}}$ (we will always use the lattice $\Sigma$ in place of $\Gamma$ in the definition of $F_{A||_{V}}$ ) given by

$$
g_{\varphi}(\gamma)=\left(\min I_{0}+u_{i_{0}}, \min I_{1}+u_{i_{1}}, \ldots\right)
$$

where $I_{k}$ is any edge of $\Phi^{-k}(\gamma)$ of type $i_{k}$. The map $g_{\varphi}$ is called geometric realization.

Note: The map $g_{\varphi}$ depends on the choice of the $u_{i} \in \Gamma / \Sigma$, which are not uniquely defined even after the normalization.

From results of $[\mathrm{BK}]$ and $[\mathrm{BBK}]$, the map $g_{\varphi}$ is boundedly finite-to-one and almost everywhere $m$-to- 1 , where $m$ is the coincidence rank of $\varphi$, and the tiling flow on $\mathcal{T}_{\varphi}$ has pure discrete spectrum if and only if $m=1$. Moreover, $g_{\varphi}$ is optimal in the sense that any other factoring of $\Phi$ onto a solenoidal shift factors through $g_{\varphi}\left({ }^{2}\right)$.

Geometric realization expresses the underlying solenoidal nature of Pisot tiling spaces. But tiling spaces, unlike solenoids, are not homogeneous. Asymptotic tilings are one kind of inhomogeneity collapsed out by geometric realization. We say the tilings $\gamma, \gamma^{\prime} \in \mathcal{T}_{\varphi}$ are forward (backward, resp.) asymptotic provided $d\left(\gamma-t, \gamma^{\prime}-t\right) \rightarrow 0$ as $t \rightarrow \infty\left(-\infty\right.$, resp.) $\left({ }^{3}\right)$. If $\gamma$ and $\gamma^{\prime}$ are asymptotic, where $\gamma \neq \gamma^{\prime}$, there is a unique $t$ so that $\gamma-t$ and $\gamma^{\prime}-t$ are periodic under inflation and substitution. Moreover, the set $\mathcal{C}_{\varphi}$ consisting of those tilings $\gamma \in \mathcal{T}_{\varphi}$ for which there is a tiling $\gamma^{\prime} \neq \gamma$ with $\gamma$ and $\gamma^{\prime}$ asymptotic and $\Phi$-periodic is finite and nonempty (see [BD1]). We will call the elements of $\mathcal{C}_{\varphi}$ special tilings.

Let $L:(V, \Sigma) \rightarrow\left(\mathbb{R}^{d}, \mathbb{Z}^{d}\right)$ be a linear isomorphism, and $F_{L}: V / \Sigma \rightarrow$ $\mathbb{T}^{d}:=\mathbb{R}^{d} / \mathbb{Z}^{d}$ the induced isomorphism. Also, let $M=M_{\varphi}$ denote the integer matrix representing the linear isomorphism $\left.L \circ A\right|_{V} \circ L^{-1}$ in the standard basis, and let $F_{M}: \mathbb{T}^{d} \oslash$ denote the corresponding toral endomorphism.

The branch locus of $\mathcal{T}_{\varphi}$ is the set

$$
\operatorname{Br}(\varphi):=\left(F_{L} \circ \pi_{0} \circ g_{\varphi}\right)\left(\mathcal{C}_{\varphi}\right) \subset \mathbb{T}^{d},
$$

$\left({ }^{2}\right)$ For the translation flow on $\mathcal{T}_{\varphi}$, the Kronecker flow, $\left(z_{0}, z_{1}, \ldots\right) \mapsto\left(z_{0}-t \omega\right.$, $\left.z_{1}-t \lambda^{-1} \omega, \ldots\right)$ on $\lim _{\longleftarrow} F_{A \mid V}$ is the maximal equicontinuous factor.

$\left({ }^{3}\right)$ There is a weakening of asymptoticity, called regional proximality, with the property that $g_{\varphi}(\gamma)=g_{\varphi}\left(\gamma^{\prime}\right)$ if and only if $\gamma$ and $\gamma^{\prime}$ are regionally proximal (see [Aus]). 
where $\pi_{0}: \lim _{A \mid V} \rightarrow V / \Sigma$ is projection onto the 0 th coordinate. Note that $F_{M}(\operatorname{Br}(\varphi))=\operatorname{Br}(\varphi)$. Also note that $\operatorname{Br}(\varphi)$ depends not only on $\varphi$ but also on the choice of $\left\{u_{i}\right\}$ in the definitions of $g_{\varphi}$ and the linear isomorphism $L$ made in the construction of $g_{\varphi}$. Our main theorem is that this dependence is limited.

Theorem 1. Suppose that $\varphi$ and $\psi$ are primitive, aperiodic, Pisot substitutions whose tiling spaces are homeomorphic. Then there are $d \times d$ integer matrices $S, T$, and $m_{0}, m_{1} \in \mathbb{N}$ such that $M_{\varphi}^{m_{0}}=S T, M_{\psi}^{m_{1}}=T S$, and translations $\tau_{0}, \tau_{1}$ on $\mathbb{T}^{d}$ such that

$$
\operatorname{Br}(\psi)=\left(\tau_{0} \circ F_{T}\right)(\operatorname{Br}(\varphi)) \text { and } \operatorname{Br}(\varphi)=\left(\tau_{1} \circ F_{S}\right)(\operatorname{Br}(\psi)) .
$$

In case $\lambda$ is a Pisot unit, that is, $\operatorname{det}\left(\left.A\right|_{V}\right)= \pm 1$, calculations are simplified.

Corollary 2. Suppose that $\varphi$ and $\psi$ are primitive and aperiodic Pisot substitutions with $\lambda_{\varphi}$ a Pisot unit of degree $d$, and that $\mathcal{T}_{\varphi}$ is homeomorphic with $\mathcal{T}_{\psi}$. Then there are $a T \in \mathrm{GL}(d, \mathbb{Z})$ and a translation $\tau$ on $\mathbb{T}^{d}$ such that

$$
\operatorname{Br}(\psi)=\tau \circ F_{T}(\operatorname{Br}(\varphi)) .
$$

Before proving the theorem, we look at a few examples.

\section{Examples}

EXAmple 1. Consider the substitution

$$
\varphi:\left\{\begin{array}{l}
1 \rightarrow 121 \\
2 \rightarrow 312 \\
3 \rightarrow 213
\end{array}\right.
$$

In this instance,

$$
A=\left(\begin{array}{lll}
2 & 1 & 1 \\
1 & 1 & 1 \\
0 & 1 & 1
\end{array}\right), \quad \lambda=3 \quad \text { and } \quad V=\mathbb{E}^{u}=\left\{t\left(\begin{array}{l}
3 \\
2 \\
1
\end{array}\right): t \in \mathbb{R}\right\}
$$

We have

$$
v_{i}=\operatorname{pr}_{V} e_{i}=\frac{1}{6}\left(\begin{array}{l}
3 \\
2 \\
1
\end{array}\right),
$$

which we denote by $v$, so that $\Gamma=\mathbb{Z} v$. Now consider the strand $\gamma$, fixed under $\Phi$,

$$
\gamma=\left\{\ldots, \sigma_{1}-2 v, \sigma_{2}-v, \sigma_{1}, \sigma_{2}+v, \sigma_{1}+2 v, \ldots\right\} .
$$

That is, $\gamma$ follows the pattern of the fixed word $\cdots 312.121 \cdots$ of $\varphi$. Clearly, if $I$ and $I^{\prime}$ are two edges of $\gamma$ of type 1 , then $\min I^{\prime}-\min I$ is of the form 
$2 k v$, where $k \in \mathbb{Z}$ (and $k=1$ occurs). Thus

$$
\Sigma=\left\langle\bigcup_{k \in \mathbb{Z}}\left(\left.A\right|_{V}\right)^{k} \Theta(1)\right\rangle \cap \Gamma=\left\langle\bigcup_{k \in \mathbb{Z}}\left(3^{k}\right)(2 v)\right\rangle \cap \Gamma=2 \Gamma=2 \mathbb{Z} v .
$$

The transition vectors are $(\bmod \Sigma) w_{21}=v, w_{31}=v$, and $w_{23}=0$. Choosing $u_{1}=0, u_{2}=v, u_{3}=v$, we have $w_{i j}=u_{j}-u_{i}$ and $A u_{i}=3 u_{i}=u_{i^{\prime}}(\bmod \Sigma)$, where $\varphi(i)=i^{\prime} \cdots$. Finally, we map $V / \Sigma$ onto $\mathbb{R} / \mathbb{Z}=\mathbb{T}^{1}$ by $F_{L}$, where $L: V \rightarrow \mathbb{R}$ is defined by $L(t v)=(t / 2) v$. Then geometric realization of $\mathcal{T}_{\varphi}$ onto a 3 -adic solenoid is given by $g_{\varphi}(\gamma)=\left(\min I_{0}-u_{i_{0}}, \min I_{1}-u_{i_{1}}, \ldots\right)$, where $I_{k}$ is an edge of $\Phi^{-k}(\gamma)$ of type $i_{k}$ and min $I_{k}$ is taken mod $\Sigma$. The branch locus of $\varphi$ is $\left(F_{L} \circ \pi_{0} \circ g_{\varphi}\right)\left(C_{\varphi}\right)$.

There are procedures for finding asymptotic tilings described in [BD1] and $[\mathrm{BDH}]$, and an applet for this purpose can be found in [E1]. For this $\varphi$, there is a single pair of backward asymptotic tilings (of period 2 under $\Phi$ ) and a single pair of forward asymptotic tilings (fixed by $\Phi$ ) in $\mathcal{C}_{\varphi}=\left\{\alpha, \alpha^{\prime}, \beta, \beta^{\prime}\right\}$, where

$$
\alpha:=\left\{\ldots, \sigma_{2}-v, \sigma_{1}, \sigma_{2}+v, \sigma_{1}+2 v, \ldots\right\}
$$

follows the pattern $\cdots 312.121 \cdots$,

$$
\alpha^{\prime}:=\left\{\ldots, \sigma_{3}-v, \sigma_{1}, \sigma_{2}+v, \sigma_{1}+2 v, \ldots\right\}
$$

follows the pattern $\cdots 213.121 \cdots$,

$$
\beta:=\left\{\ldots, \sigma_{1}-v, \sigma_{3}, \sigma_{1}+v, \sigma_{2}+2 v, \ldots\right\}
$$

follows the pattern $\cdots 121.312 \cdots$, and

$$
\beta^{\prime}:=\left\{\ldots, \sigma_{1}-v, \sigma_{2}, \sigma_{1}+v, \sigma_{3}+2 v, \ldots\right\}
$$

follows the pattern $\cdots 121.213 \cdots$. Thus $\operatorname{Br}(\varphi)=\{0+\mathbb{Z}, 1 / 2+\mathbb{Z}\}$. The periodic forward asymptotic tilings are "halfway around" the tiling space from the periodic backward asymptotic tilings.

Note: One can show that $\Gamma / \Sigma \simeq \mathbb{Z} / h \mathbb{Z}$, where $h$ is the height of the substitution $\varphi$ (see [D]), if $\varphi$ is constant length (as in Example 1) or, more generally, $\varphi$ is Pisot of degree 1 . In this sense, the group $\Gamma / \Sigma$ is an extension of the notion of the height of a constant length substitution to the higher degree setting.

EXAMPLE 2. We will show that two particular tiling spaces are not homeomorphic by considering their branch loci. The Matsumoto $K_{0}$-group $([\mathrm{M}])$ and the crossing group ([BSw1]) do not distinguish these spaces. Also, we have not been able to distinguish these spaces by the generalized BowenFranks trace ([BSw1]) or the proximality structures described in [BD2]. The (unordered) augmented cohomology groups of these spaces are isomorphic, but one can show that the Pisot parts of the augmented dimension groups (see $\S 5$ ) are not order isomorphic. 
Consider the substitutions

$$
\varphi:\left\{\begin{array}{l}
a \rightarrow a a a^{12} b^{16} a a \\
b \rightarrow a b a^{14} b^{14} b a
\end{array} \text { and } \quad \psi:\left\{\begin{array}{l}
a \rightarrow a a a a^{11} b^{16} a a \\
b \rightarrow a a b a^{13} b^{14} b a .
\end{array}\right.\right.
$$

Here

$$
\begin{gathered}
A_{\varphi}=A_{\psi}=\left(\begin{array}{ll}
16 & 16 \\
16 & 16
\end{array}\right), \quad v_{\varphi}=v_{\psi}=\mathbb{E}^{u}=\left\{t\left(\begin{array}{l}
1 \\
1
\end{array}\right): t \in \mathbb{R}\right\}, \\
v_{1}=v_{2}=v=\left(\begin{array}{c}
1 / 2 \\
1 / 2
\end{array}\right)
\end{gathered}
$$

for both $\varphi$ and $\psi$, and $\Sigma_{\varphi}=\Gamma_{\varphi}=\Gamma_{\psi}=\Sigma_{\psi}=\mathbb{Z} v$. In geometric realization all the $u_{i}$ can be taken to be 0 . Choosing $L: t v \mapsto t$, one gets $\operatorname{Br}(\varphi)=$ $\{1 / 31+\mathbb{Z}, 30 / 31+\mathbb{Z}\}, \operatorname{Br}(\psi)=\{2 / 31+\mathbb{Z}, 30 / 31+\mathbb{Z}\}$, and $M=M_{\varphi}=$ $M_{\psi}=(32)$. So, if $\mathcal{T}_{\varphi}$ is homeomorphic with $\mathcal{T}_{\psi}$, there must be $S=(s)$ and $T=(t)$, where $s, t \in \mathbb{Z}$, an $m \in \mathbb{N}$ such that $M^{m}=S T$, and a translation $\tau$ on $\mathbb{T}^{1}=\mathbb{R} / \mathbb{Z}$ such that $\tau \circ F_{T} \operatorname{Br}(\varphi)=\operatorname{Br}(\psi)$. The only possibility for $t$ is $\pm 2^{k}$, where $k \in \mathbb{N}$. Then $\tau\left(\left( \pm 2^{k}\right)\{1 / 31,30 / 31\}\right)=\{2 / 31,30 / 31\}(\bmod 1)$ implies that $\left( \pm 2^{k}\right)(29 / 31)=(28 / 31)(\bmod 1)$, hence that $\left( \pm 2^{k}\right)(29)=28$ $(\bmod 31)$, or $\pm 2^{k+1}=3(\bmod 31)$, which is not the case for any $k \in \mathbb{N}$. It follows from Theorem 1 that $\mathcal{T}_{\varphi}$ and $\mathcal{T}_{\psi}$ are not homeomorphic.

EXAmple 3. Define

$$
\varphi:\left\{\begin{array}{l}
1 \rightarrow 1122111 \\
2 \rightarrow 1211111221
\end{array} \text { and } \psi:\left\{\begin{array}{l}
1 \rightarrow 1121211 \\
2 \rightarrow 1211112211
\end{array}\right.\right.
$$

Then $A=A_{\varphi}=A_{\psi}=\left(\begin{array}{ll}5 & 7 \\ 2 & 3\end{array}\right)$, so $\lambda=\lambda_{\varphi}=\lambda_{\psi}$ is a Pisot unit and Corollary 2 applies. As $\lambda$ has degree $2, \Sigma_{\varphi}=\Sigma_{\psi}=\mathbb{Z}^{2}$. Taking $L$ to be the identity map, geometrical realization for both $\mathcal{T}_{\varphi}$ and $\mathcal{T}_{\psi}$ takes the form $\gamma \mapsto \min I$ $\left(\bmod \mathbb{Z}^{2}\right)$, where this last $I$ is an edge of $\gamma$. Each of $\varphi$ and $\psi$ has a pair of backward and a pair of forward asymptotic tilings, fixed under inflation and substitution. Consider, for example, the tilings for $\varphi$ following the patterns

$$
\cdots 11221111122111 \cdots \text { and } \cdots 11221111211111221 \cdots \text {, }
$$

where the dot in each word above indicates the position of the origin. To locate the corresponding strands (call them $\gamma_{1}$ and $\gamma_{2}$ ), we seek $x \in \mathbb{R}^{2}$ such that

$$
\Phi\left(\sigma_{1}+x\right)=\left\{\sigma_{1}+x-e_{1}, \sigma_{1}+x, \sigma_{2}+x+e_{1}, \ldots\right\}
$$

or, equivalently,

$$
\Phi\left(\sigma_{2}+x\right)=\left\{\sigma_{1}+x-e_{1}, \sigma_{2}+x, \sigma_{1}+x+e_{2}, \ldots\right\} .
$$

Since

$$
\Phi\left(\sigma_{1}+x\right)=\left\{\sigma_{1}+A x, \sigma_{1}+A x+e_{1}, \ldots\right\}
$$


we must have $A x=x-e_{1}$. This yields $x=\left(\begin{array}{c}1 / 3 \\ -1 / 3\end{array}\right)$, a vertex of both $\gamma_{1}$ and $\gamma_{2}$. Thus

$$
g_{\varphi}\left(\gamma_{1}\right)=g_{\varphi}\left(\gamma_{2}\right)=\left(\begin{array}{c}
1 / 3 \\
-1 / 3
\end{array}\right)=\left(\begin{array}{c}
1 / 3 \\
2 / 3
\end{array}\right)\left(\bmod \mathbb{Z}^{2}\right) \in \operatorname{Br}(\varphi) .
$$

Similar calculations yield

$$
\begin{aligned}
& \operatorname{Br}(\varphi)=\left\{\left(\begin{array}{l}
1 / 3 \\
2 / 3
\end{array}\right)+\mathbb{Z}^{2},\left(\begin{array}{c}
2 / 3 \\
1 / 3
\end{array}\right)+\mathbb{Z}^{2}\right\}, \\
& \operatorname{Br}(\psi)=\left\{\left(\begin{array}{c}
1 / 3 \\
2 / 3
\end{array}\right)+\mathbb{Z}^{2},\left(\begin{array}{c}
1 / 2 \\
0
\end{array}\right)+\mathbb{Z}^{2}\right\} .
\end{aligned}
$$

Were $\mathcal{T}_{\varphi}$ and $\mathcal{T}_{\psi}$ homeomorphic, there would be a $T \in \mathrm{GL}(2, \mathbb{Z})$ and a translation $\tau$ on $\mathbb{T}^{2}$ so that $\left(\tau \circ F_{T}\right)(\operatorname{Br}(\varphi))=\operatorname{Br}(\psi)$. In other words,

$$
F_{T}\left[\left(\begin{array}{l}
1 / 3 \\
2 / 3
\end{array}\right)-\left(\begin{array}{l}
2 / 3 \\
1 / 3
\end{array}\right)+\mathbb{Z}^{2}\right]= \pm\left[\left(\begin{array}{l}
1 / 3 \\
2 / 3
\end{array}\right)-\left(\begin{array}{c}
1 / 2 \\
0
\end{array}\right)+\mathbb{Z}^{2}\right] \text {. }
$$

That is, $T \in \mathrm{GL}(2, \mathbb{Z})$ would satisfy

$$
\frac{1}{3} T\left(\begin{array}{l}
1 \\
2
\end{array}\right)-\frac{1}{6}\left(\begin{array}{l}
1 \\
2
\end{array}\right) \in \mathbb{Z}^{2}
$$

Since this is not possible, Corollary 2 implies that $\mathcal{T}_{\varphi}$ and $\mathcal{T}_{\psi}$ are not homeomorphic.

Example 4. Consider

$$
\varphi:=\left\{\begin{array}{l}
a \rightarrow a a a^{2} b^{4} c a c \\
b \rightarrow a b a^{7} b^{6} c^{5} b b c \\
c \rightarrow a a b a^{6} b^{6} c^{3} c b c
\end{array} \quad \text { and } \quad \psi:=\left\{\begin{array}{l}
a \rightarrow a a a b^{4} c a a c \\
b \rightarrow a a b a^{5} b^{5} c^{5} b b b a c \\
c \rightarrow a a b b a a^{4} b^{4} c^{3} c b b a c
\end{array}\right.\right.
$$

with abelianization

$$
A=\left(\begin{array}{lll}
5 & 8 & 8 \\
4 & 9 & 8 \\
2 & 6 & 5
\end{array}\right)
$$

The projection along $\left(\begin{array}{c}2 \\ 0 \\ -1\end{array}\right)$ onto the Pisot subspace $V$ is given by

$$
\operatorname{pr}_{V}=\left(\begin{array}{ccc}
0 & 1 & 0 \\
0 & 1 & 0 \\
1 / 2 & -1 / 2 & 1
\end{array}\right)
$$

In this case the lattices $\Gamma$ and $\Sigma$ are equal, since return vectors span $\Gamma$ over $\mathbb{Z}$ (the repeated letters $a a, b b$, and $c c$ yield return vectors $v_{i}=\operatorname{pr}_{V}\left(e_{i}\right)$, 
$i=1,2,3)$. The set

$$
\left\{v_{1}, v_{1}+v_{2}\right\}=\left\{\left(\begin{array}{c}
0 \\
0 \\
1 / 2
\end{array}\right),\left(\begin{array}{l}
1 \\
1 \\
0
\end{array}\right)\right\}
$$

is a basis for $\Sigma$ over $\mathbb{Z}$. In these coordinates, $\left.A\right|_{V}$ takes the form $M=\left(\begin{array}{ll}5 & 16 \\ 4 & 13\end{array}\right)$.

There are one pair of fixed backward asymptotic tilings and two pairs of fixed forward asymptotic tilings for both $\varphi$ and $\psi$. We solve the equations for the branch locus points in a manner similar to the foregoing examples:

\begin{tabular}{c|c}
\hline $\operatorname{Br}(\varphi)\left(\bmod \mathbb{Z}^{2}\right)$ & $\operatorname{Br}(\psi)\left(\bmod \mathbb{Z}^{2}\right)$ \\
\hline$(M-I)^{-1}\left(\begin{array}{r}-1 \\
0\end{array}\right)=\left(\begin{array}{l}3 / 4 \\
3 / 4\end{array}\right)$ & $(M-I)^{-1}\left(\begin{array}{r}-2 \\
0\end{array}\right)=\left(\begin{array}{l}1 / 2 \\
1 / 2\end{array}\right)$ \\
$(M-I)^{-1}\left(\begin{array}{l}2 \\
0\end{array}\right)=\left(\begin{array}{c}1 / 2 \\
1 / 2\end{array}\right)$ & $(M-I)^{-1}\left(\begin{array}{l}3 \\
0\end{array}\right)=\left(\begin{array}{l}3 / 4 \\
3 / 4\end{array}\right)$ \\
$(M-I)^{-1}\left(\begin{array}{l}1 \\
1\end{array}\right)=\left(\begin{array}{c}1 / 4 \\
0\end{array}\right)$ & $(M-I)^{-1}\left(\begin{array}{l}1 \\
2\end{array}\right)=\left(\begin{array}{l}1 / 4 \\
3 / 4\end{array}\right)$ \\
\hline
\end{tabular}

There are infinitely many different triangles on the 2-torus having a given set of three points as vertices. In case the vertices are the points of $\operatorname{Br}(\varphi)$, we write the general edge vectors, up to integer translation in the lift, as $(1 / 4+p, 1 / 2+q)$ and $(1 / 4+r, 1 / 4+s)$. The area has the form

$$
\left|\frac{1}{16}-\frac{1}{8}+\frac{1}{4}(p+s+4 p s-q-2 r-4 r q)\right| .
$$

Since $p, q, r, s$ are integers, the minimum area is $\frac{1}{16}$ (e.g. $p=q=r=s=0$ ). The argument for $\operatorname{Br}(\psi)$ is similar, but the minimum area is $\frac{1}{8}$. Were $\mathcal{T}_{\varphi}$ and $\mathcal{T}_{\psi}$ homeomorphic, the map $\tau \circ T$ of Corollary 6 would preserve the areas of these triangles. We conclude they are not homeomorphic.

EXAMPLE 5. The pair

$$
\varphi:=\left\{\begin{array}{l}
a \rightarrow a a b b c a c \\
b \rightarrow a b a b c c b c c \\
c \rightarrow a a a^{4} b^{6} c^{8}
\end{array} \quad \text { and } \quad \psi:=\left\{\begin{array}{l}
a \rightarrow c a a b b a c \\
b \rightarrow c a b a b c b c c \\
c \rightarrow c a a a^{4} b^{6} c^{7}
\end{array}\right.\right.
$$

provides an example of substitutions $\varphi$ and $\psi$ such that the Perron-Frobenius eigenvalue $\lambda_{\varphi}=\lambda_{\psi}$ is Pisot but not a unit. The reader can check that the branch locus $\operatorname{Br}(\psi)$ is colinear, whereas $\operatorname{Br}(\varphi)$ is not. By Theorem $1, \mathcal{T}_{\varphi}$ and $\mathcal{T}_{\psi}$ are not homeomorphic.

4. Proof of Theorem 1. In [BSw2] it is shown that if two substitution tiling spaces are homeomorphic then there is a "linear" homeomorphism 
between the tiling spaces that conjugates some powers of their inflation and substitution homeomorphisms. This linear homeomorphism necessarily takes special tilings to special tilings. The proof of Theorem 1 will proceed by pushing this linear homeomorphism down to the level of geometric realization. This will be accomplished by representing the linear homeomorphism as a shift equivalence between certain rewritings of (powers of) the substitutions involved. The shift equivalence will be defined by a pair of morphisms that induce maps on strands; abelianization of these morphisms produces the matrices $S$ and $T$ of the theorem.

Suppose that $\varphi$ is a primitive and aperiodic substitution with language

$$
\mathcal{L}_{\varphi}:=\left\{w \in \mathcal{A}^{*}: w \text { is a factor of } \varphi^{n}(i) \text { for some } i \in \mathcal{A}, n \in \mathbb{N}\right\}
$$

and allowed bi-infinite words

$$
X_{\varphi}:=\left\{\cdots x_{-1} x_{0} x_{1} \cdots: x_{n} \in \mathcal{A}, x_{n} \cdots x_{n+k} \in \mathcal{L}_{\varphi} \text { for } n \in \mathbb{Z}, k \in \mathbb{N}\right\} .
$$

Suppose that the finite subset $W=\left\{w_{1}, \ldots, w_{k}\right\} \subset \mathcal{L}_{\varphi}$ has the properties that (1) each element of $X_{\varphi}$ can be uniquely factored as a product of elements of $W$, and (2) for each $w_{i} \in W, \varphi\left(w_{i}\right)$ can be uniquely factored as a product of elements of $W$. We may then define a substitution $\varphi^{\prime}:\{1, \ldots, k\} \rightarrow\{1, \ldots, k\}^{*}$ by the rule $\varphi^{\prime}(i)=i_{1} \cdots i_{l}$ provided $\varphi\left(w_{i}\right)=w_{i_{1}} \cdots w_{i_{l}}$. We will call such a $\varphi^{\prime}$ a rewriting of $\varphi$, and the morphism $\rho:\{1, \ldots, k\} \rightarrow \mathcal{A}^{*}$ given by $\rho(i)=w_{i}$ the associated rewriting morphism. Note that $\rho \circ \varphi^{\prime}=\varphi \circ \rho$. It is not hard to see that $\varphi^{\prime}$ is also primitive and aperiodic.

Suppose now that $\varphi^{\prime}$ and $\varphi$ are substitutions and $\rho$ is a morphism with $\rho \circ \varphi^{\prime}=\varphi \circ \rho$. Then the abelianizations $A, A^{\prime}$ and $P$ of $\varphi, \varphi^{\prime}$ and $\rho$, resp., satisfy $P A^{\prime}=A P$. Consequently, if $w$ and $w^{\prime}$ are positive right PerronFrobenius eigenvectors of $A$ and $A^{\prime}$, resp., then $P w^{\prime}=a w$ for some $a>0$, and $A$ and $A^{\prime}$ have the same Perron-Frobenius eigenvalue. Thus $\varphi^{\prime}$ is Pisot if and only if $\varphi$ is. In this case, let $V$ and $V^{\prime}$ be the invariant Pisot subspaces (with rational bases) corresponding to $A$ and $A^{\prime}$, resp. Since $w \in P V^{\prime} \cap V$, $P V^{\prime} \cap V$ is a nontrivial rational subspace of $V$. The characteristic polynomial of $\left.A\right|_{V}$ is irreducible, and $P V^{\prime} \cap V$ is $A$-invariant, which implies that $P V^{\prime} \cap$ $V=V$ and $V \subset P V^{\prime}$. But $\operatorname{dim} V=d=\operatorname{dim} V^{\prime}$, hence $P\left(V^{\prime}\right)=V$ and $\left.P\right|_{V^{\prime}}: V^{\prime} \rightarrow V$ is invertible. Furthermore, if $\varphi$ and $\varphi^{\prime}$ are Pisot, there is a natural continuous surjection $\bar{\rho}: \mathcal{T}_{\varphi^{\prime}} \rightarrow \mathcal{T}_{\varphi}$ that conjugates $\Phi^{\prime}$ with $\Phi$, defined as follows: Given an edge $I=\sigma_{i}^{\prime}+x$ in $V^{\prime}$, with $\rho(i)=i_{1} \cdots i_{l}$ and $v_{j}:=\pi_{V} e_{j}$, let $\bar{\rho}(I)$ denote the finite strand in $V$ defined by

$$
\bar{\rho}(I):=\left\{\sigma_{i_{1}}+P x, \sigma_{i_{2}}+P x+v_{i_{1}}, \ldots, \sigma_{i_{l}}+P x+v_{i_{1}}+\cdots+v_{i_{l-1}}\right\} .
$$

If $\gamma^{\prime}=\left\{\sigma_{i_{k}}^{\prime}+x_{k}: k \in \mathbb{Z}\right\}$ is any strand in $V^{\prime}$, let $\bar{\rho}\left(\gamma^{\prime}\right)$ denote the strand in 
$V$ made up of the finite substrands $\bar{\rho}\left(\sigma_{i_{k}}+x_{k}\right)$ given by

$$
\bar{\rho}\left(\gamma^{\prime}\right)=\bigcup_{k \in \mathbb{Z}} \bar{\rho}\left(\sigma_{i_{k}}^{\prime}+x_{k}\right) .
$$

Observe that $\bar{\rho} \circ \Phi^{\prime}=\Phi \circ \bar{\rho}$. Since $P \mathbb{E}_{\varphi^{\prime}}^{u}=\mathbb{E}_{\varphi}^{u}$, there is an $R>0$ such that $\bar{\rho}\left(\mathcal{T}_{\varphi^{\prime}}\right) \subset \mathcal{F}_{\varphi}^{R}$. Thus if $\gamma^{\prime} \in \mathcal{T}_{\varphi^{\prime}}$, then

$$
\bar{\rho}\left(\gamma^{\prime}\right)=\bar{\rho}\left(\left(\Phi^{\prime}\right)^{n}\left(\Phi^{\prime}\right)^{-n}\left(\gamma^{\prime}\right)\right)=\Phi^{n}\left(\bar{\rho}\left(\left(\Phi^{\prime}\right)^{-n}\left(\gamma^{\prime}\right)\right) \in \Phi^{n} \mathcal{F}_{\varphi}^{R}\right.
$$

for all $n \in \mathbb{N}$, so

$$
\bar{\rho}\left(\gamma^{\prime}\right) \in \bigcap_{n \in \mathbb{N}} \Phi^{n}\left(\mathcal{F}_{\varphi}^{R}\right)=\mathcal{T}_{\varphi}
$$

Continuity of $\bar{\rho}$ is clear, and from the fact that $\bar{\rho}\left(\gamma^{\prime}-t\right)=\bar{\rho}\left(\gamma^{\prime}\right)-a t$ and minimality of the tiling flow, it follows that $\bar{\rho}: \mathcal{T}_{\varphi^{\prime}} \rightarrow \mathcal{T}_{\varphi}$ is surjective.

Suppose that $V$ and $V^{\prime}$ are finite-dimensional real vector spaces and $\Sigma \subset V, \Sigma^{\prime} \subset V^{\prime}$ are lattices. By a map $T:(V, \Sigma) \rightarrow\left(V^{\prime}, \Sigma^{\prime}\right)$, we will mean a linear transformation $T: V \rightarrow V^{\prime}$ with $T(\Sigma) \subset \Sigma^{\prime}$. Two maps $A$ : $(V, \Sigma) \rightarrow(V, \Sigma)$ and $A^{\prime}:\left(V^{\prime}, \Sigma^{\prime}\right) \rightarrow\left(V^{\prime}, \Sigma^{\prime}\right)$ are shift-equivalent, $A \sim_{\mathrm{SE}} A^{\prime}$, provided there are maps $T:(V, \Sigma) \rightarrow\left(V^{\prime}, \Sigma^{\prime}\right)$ and $S:\left(V^{\prime}, \Sigma^{\prime}\right) \rightarrow(V, \Sigma)$ and natural numbers $m, n$ such that $A^{m}=S T,\left(A^{\prime}\right)^{n}=T S, A^{\prime} T=T A$, and $S A^{\prime}=A S ; S$ and $T$ express the shift equivalence. The relation $\sim_{\mathrm{SE}}$ is an equivalence relation, and if $A \sim_{\mathrm{SE}} A^{\prime}$, then $A^{k} \sim_{\mathrm{SE}}\left(A^{\prime}\right)^{k}$ for all $k \in \mathbb{N}$. Furthermore, if $\hat{F}_{T}: \lim _{\longleftarrow} F_{A} \rightarrow \lim _{\longleftarrow} F_{A^{\prime}}$ is defined by

$$
\hat{F}_{T}\left(x_{1}+\Sigma, x_{2}+\Sigma, \ldots\right)=\left(T x_{1}+\Sigma^{\prime}, T x_{2}+\Sigma^{\prime}, \ldots\right)
$$

(and $\hat{F}_{S}, \hat{F}_{A^{\prime}}$ and $\hat{F}_{A}$ are defined similarly), and if $\Sigma$ and $\Sigma^{\prime}$ are co-compact, then $\hat{F}_{T}$ and $\hat{F}_{S}$ are topological isomorphisms of the solenoids that conjugate the shifts $\hat{F}_{A}$ and $\hat{F}_{A^{\prime}}$.

Lemma 3. Suppose that $\varphi$ and $\varphi^{\prime}$ are Pisot substitutions and that either

(a) $\varphi^{\prime}$ is a rewriting of $\varphi$ with rewriting morphism $\rho$, or

(b) there are morphisms $\alpha, \beta$ such that $\varphi=\alpha \circ \beta$ and $\varphi^{\prime}=\beta \circ \alpha$.

Let $A, V$ and $\Sigma\left(A^{\prime}, V^{\prime}\right.$ and $\Sigma^{\prime}$, resp. $)$ be the abelianization, invariant Pisot subspace and return lattice for $\varphi\left(\varphi^{\prime}\right.$, resp.). Then, in the case of (a),

(1a) $\bar{\rho}: \mathcal{T}_{\varphi^{\prime}} \rightarrow \mathcal{T}_{\varphi}$ is a homeomorphism,

while in the case of $(\mathrm{b})$,

(1b) $\bar{\alpha}: \mathcal{T}_{\varphi^{\prime}} \rightarrow \mathcal{T}_{\varphi}$ and $\bar{\beta}: \mathcal{T}_{\varphi} \rightarrow \mathcal{T}_{\varphi^{\prime}}$ are homeomorphisms. Also,

(2) the maps $\left.A\right|_{V}:(V, \Sigma) \rightarrow(V, \Sigma)$ and $\left.A^{\prime}\right|_{V^{\prime}}:\left(V^{\prime}, \Sigma^{\prime}\right) \rightarrow\left(V^{\prime}, \Sigma^{\prime}\right)$ are shift equivalent. 
Furthermore, if $g_{\varphi}: \mathcal{T}_{\varphi} \rightarrow \lim _{\longleftarrow} F_{\left.A\right|_{V}}$ and $g_{\varphi^{\prime}}: \mathcal{T}_{\varphi^{\prime}} \rightarrow \lim _{\longleftarrow} F_{\left.A^{\prime}\right|_{V^{\prime}}}$ are geometric realizations onto the natural solenoids, and $T: \overleftarrow{(V, \Sigma) \rightarrow\left(V^{\prime}, \Sigma^{\prime}\right)}$ and $S:\left(V^{\prime}, \Sigma^{\prime}\right) \rightarrow(V, \Sigma)$ express the shift equivalence in $(2)$, then there are translations $\tau$ and $\tau^{\prime}$ on $\varliminf_{\longleftarrow} F_{\left.A\right|_{V}}$ and $\varliminf_{\longleftarrow} F_{\left.A^{\prime}\right|_{V^{\prime}}}$ such that, in the case of (a),

(3a) $g_{\varphi} \circ \bar{\rho}=\tau \circ \hat{F}_{S} \circ g_{\varphi^{\prime}}$ and $g_{\varphi^{\prime}} \circ \bar{\rho}^{-1}=\tau^{\prime} \circ \hat{F}_{T} \circ \hat{F}_{\left.A\right|_{V}}^{m} \circ g_{\varphi}$ for some $m \in \mathbb{N}$,

while in the case of $(\mathrm{b})$,

(3b) $g_{\varphi} \circ \bar{\alpha}=\tau \circ \hat{F}_{S} \circ g_{\varphi^{\prime}}$ and $g_{\varphi^{\prime}} \circ \bar{\beta}=\tau^{\prime} \circ \hat{F}_{T} \circ g_{\varphi}$.

Proof. Suppose that $\varphi^{\prime}$ is a rewriting of $\varphi$ with rewriting morphism $\rho$ having abelianization $P$, and suppose that $\gamma^{\prime}, \gamma^{\prime \prime} \in \mathcal{T}_{\varphi^{\prime}}$ are such that $\bar{\rho}\left(\gamma^{\prime}\right)=\bar{\rho}\left(\gamma^{\prime \prime}\right)$. Let $w^{\prime}$ and $w^{\prime \prime}$ be the words of $X_{\varphi^{\prime}}$ spelled out by $\gamma^{\prime}$ and $\gamma^{\prime \prime}$, where some location is chosen for the decimal point indicating the location of the 0th letter. Then $\rho\left(w^{\prime}\right)$ and $\rho\left(w^{\prime \prime}\right)$ agree, up to shift, so that $w^{\prime}$ and $w^{\prime \prime}$ must agree, up to shift, by the uniqueness of factorization in $X_{\varphi}$ in the definition of rewriting. It follows that $\gamma^{\prime}=\gamma^{\prime \prime}-t$ for some $t$. But then $\bar{\rho}\left(\gamma^{\prime \prime}\right)=\bar{\rho}\left(\gamma^{\prime}\right)=\bar{\rho}\left(\gamma^{\prime \prime}-t\right)=\bar{\rho}\left(\gamma^{\prime \prime}\right)-a t$, hence $t=0$ and $\gamma^{\prime}=\gamma^{\prime \prime}$. That is, $\bar{\rho}$ is a homeomorphism.

In case $\varphi=\alpha \circ \beta$ and $\varphi^{\prime}=\beta \circ \alpha$, we have $\bar{\alpha} \circ \bar{\beta}=\Phi$ and $\bar{\beta} \circ \bar{\alpha}=\Phi^{\prime}$, so both $\bar{\alpha}$ and $\bar{\beta}$ must be homeomorphisms.

Again, suppose that $\varphi^{\prime}$ is a rewriting of $\varphi$. If $v \in \Theta^{\prime}(i)$ is a return vector for $\varphi^{\prime}$, say $\gamma^{\prime} \in \mathcal{T}_{\varphi^{\prime}}$ has edges $I$ and $I+v$ of type $i$, then $\bar{\rho} \gamma^{\prime} \in \mathcal{T}_{\varphi}$ has edges $J$ and $J+P v$ of type $i_{1}$, where $\rho(i)=i_{1} \cdots$. Thus $P v \in \Theta\left(i_{1}\right)$ is a return vector for $\varphi$. It follows that $P \Sigma^{\prime} \subset \Sigma$.

While it is not necessarily the case that $P \Sigma^{\prime}=\Sigma$, we shall see that $A^{m} \Sigma \subset P \Sigma^{\prime}$ for some $m \in \mathbb{N}$. To this end, suppose that $I$ is an edge in $V$ of type $i$, and $I, I+v$ are edges of $\gamma \in \mathcal{T}_{\varphi}$ with $v \neq 0$. Let $\gamma^{\prime}:=\bar{\rho}^{-1}(\gamma)$, and for each $n \in \mathbb{N}$, let $\delta^{n}$ and $\eta^{n}$ be maximal substrands of $\left(\Phi^{\prime}\right)^{n}\left(\gamma^{\prime}\right)$ with the property that $\bar{\rho}\left(\delta^{n}\right) \subset \Phi^{n}(I)$ and $\bar{\rho}\left(\eta^{n}\right) \subset \Phi^{n}(I+v)$. If $x^{n}, y^{n} \in \mathcal{L}_{\varphi^{\prime}}$ are the words corresponding to $\delta^{n}, \eta^{n}$, resp., then we have the factorizations $p^{n} \rho\left(x^{n}\right) s^{n}$ and $q^{n} \rho\left(y^{n}\right) r^{n}$ of $\varphi^{n}(i)$ in which the words $p^{n}, s^{n}, r^{n}, q^{n} \in \mathcal{L}_{\varphi}$ are of bounded length. Putting a decimal point (to mark the position of the 0th coordinate) on the left of $\varphi^{n}(i)$, we may choose $n_{k} \rightarrow \infty$ and $m_{k} \in \mathbb{N}$, with $m_{k}$ on the order of $n_{k} / 2$, so that $\sigma^{m_{k}}\left(\varphi^{n_{k}}(i)\right)$ converges to a bi-infinite word $w \in X_{\varphi}$ (here $\sigma$ is the shift that takes.$a b c \cdots$ to $a . b c \cdots$ ). Let $i^{k}$ be the letter (in $\mathcal{A}=\mathcal{A}_{\varphi}$ ) immediately to the right of the decimal point in $\sigma^{m_{k}}\left(\varphi^{n_{k}}(i)\right)$, and let $x_{0}^{n_{k}}$ and $y_{0}^{n_{k}}$ denote the letters (in $\left.\mathcal{A}^{\prime}=\mathcal{A}_{\varphi^{\prime}}\right)$ of $x^{n_{k}}$ and $y^{n_{k}}$, resp., such that $\rho\left(x_{0}^{n_{k}}\right)$ and $\rho\left(y_{0}^{n_{k}}\right)$ contain (the identified occurrence of) $i^{k}$. Putting a decimal point immediately to the left of $x_{0}^{n_{k}}$ and $y_{0}^{n_{k}}$ in $x^{n_{k}}$ and $y^{n_{k}}$, resp., we may choose a subsequence $n_{k_{l}}$ so that $x^{n_{k_{l}}}$ and $y^{n_{k_{l}}}$ converge to bi-infinite words, say $x$ and $y$, resp., in $X_{\varphi^{\prime}}$. Now $\rho(x)$ and 
$\rho(y)$ give factorings of $w \in X_{\varphi}$ by the words $w_{i}=\rho(j)$ for $j \in \mathcal{A}^{\prime}$. By uniqueness, $x=y$. Thus for large $l, x_{0}^{n_{k_{l}}}=y_{0}^{n_{k_{l}}}$. Pick such an $l$, let $m=n_{k_{l}}$, $j=x_{0}^{m}=y_{0}^{m}$, and $J, J+v^{\prime}$ be the edges of the substrands $\delta^{m}, \eta^{m}$ of $\left(\Phi^{\prime}\right)^{m}\left(\gamma^{\prime}\right)$ corresponding to $x_{0}^{m}$ and $y_{0}^{m}$, resp. Then $P\left(v^{\prime}\right)=A^{m} v$. That is, given $v \in \Theta(i)$, a return vector for $\varphi$, there is $v^{\prime} \in \Theta^{\prime}(j)$, a return vector for $\varphi^{\prime}$, with $v=\left(\left.A\right|_{V}\right)^{-m} P\left(v^{\prime}\right)=P\left(\left.A^{\prime}\right|_{V^{\prime}}\right)^{-m}\left(v^{\prime}\right)$.

Now suppose that $v_{1}, \ldots, v_{d}$ is a basis for $\Sigma$, the return lattice for $\varphi$. Each $v_{j}$ is then an integer combination of elements of $\bigcup_{k \in \mathbb{Z}}\left(\left.A\right|_{V}\right)^{k} \Theta(i)$. The preceding argument shows that there is an $m \in \mathbb{N}$ such that $A^{m} v_{j}$ is in $P \Sigma^{\prime}$ for all $j=1, \ldots, d$. That is, $A^{m} \Sigma \subset P \Sigma^{\prime}$. Let $S:=\left.P\right|_{V^{\prime}}$ and $T:=$ $\left(\left.P\right|_{V^{\prime}}\right)^{-1}\left(\left.A\right|_{V}\right)^{m}$ (recall that $\left.P\right|_{V^{\prime}}$ is invertible because $\varphi$ and $\varphi^{\prime}$ are Pisot). We have $S:\left(V^{\prime}, \Sigma^{\prime}\right) \rightarrow(V, \Sigma), T:(V, \Sigma) \rightarrow\left(V^{\prime}, \Sigma^{\prime}\right),\left.A\right|_{V} S=\left.S A^{\prime}\right|_{V^{\prime}}$, $\left.T A\right|_{V}=\left.A^{\prime}\right|_{V^{\prime}} T, S T=\left(\left.A\right|_{V}\right)^{m}$, and $T S=\left(\left.A^{\prime}\right|_{V^{\prime}}\right)^{m}$.

Still in the rewriting case, suppose that $g_{\varphi}: \mathcal{T}_{\varphi} \rightarrow \lim F_{\left.A\right|_{V}}$ and $g_{\varphi^{\prime}}$ : $\mathcal{T}_{\varphi^{\prime}} \rightarrow \underset{\lim }{\longleftarrow} F_{\left.A^{\prime}\right|_{V^{\prime}}}$ are geometric realizations onto the natural solenoids defined by the selection of $\left\{u_{i}\right\}$ in $\Gamma / \Sigma$ and $\left\{u_{i}^{\prime}\right\}$ in $\Gamma^{\prime} / \Sigma^{\prime}$, resp. (see the definition of geometric realization in $\S 2$ ). Let $\left\{w_{i j}\right\} \subset \Gamma / \Sigma$ and $\left\{w_{i j}^{\prime}\right\} \subset \Gamma^{\prime} / \Sigma^{\prime}$ denote the corresponding transition vectors (so $w_{i j}=u_{j}-u_{i}$ and $w_{i j}^{\prime}=u_{j}^{\prime}-u_{i}^{\prime}$ for all $i, j)$. Let $\gamma^{\prime} \in \mathcal{T}_{\varphi^{\prime}}$ have edge $I$ of type $i$. Then $g_{\varphi^{\prime}}\left(\gamma^{\prime}\right)$ has 0th coordinate

$$
\left(g_{\varphi^{\prime}}\left(\gamma^{\prime}\right)\right)_{0}=\left(\min I+\Sigma^{\prime}\right)-u_{i}^{\prime}
$$

in $V^{\prime} / \Sigma^{\prime}$. The strand $\bar{\rho}\left(\gamma^{\prime}\right)$ in $\mathcal{T}_{\varphi}$ has an edge $J$ of type $i_{1}$, where $\rho(i)=i_{1} \cdots$, with $\min J=P \min I$. Thus

$$
\left(g_{\varphi}\left(\bar{\rho}\left(\gamma^{\prime}\right)\right)\right)_{0}=((P \min I)+\Sigma)-u_{i_{1}} .
$$

We have

$$
F_{P}\left(\left(g_{\varphi^{\prime}}\left(\gamma^{\prime}\right)\right)_{0}\right)=((P \min I)+\Sigma)-F_{P} u_{i}^{\prime}=\left(g_{\varphi}\left(\bar{\rho}\left(\gamma^{\prime}\right)\right)\right)_{0}+u_{i_{1}}-F_{P} u_{i}^{\prime} .
$$

We claim that $u_{i_{1}}-F_{P} u_{i}^{\prime}$ is independent of $i \in \mathcal{A}^{\prime}$. Indeed,

$$
\begin{aligned}
\left(u_{i_{1}}-F_{P} u_{i}^{\prime}\right)-\left(u_{j_{1}}-F_{P} u_{j}^{\prime}\right) & =\left(u_{i_{1}}-u_{j_{1}}\right)-F_{P}\left(u_{i}^{\prime}-u_{j}^{\prime}\right) \\
& =w_{j_{1} i_{1}}-F_{P}\left(w_{j i}^{\prime}\right) .
\end{aligned}
$$

But if $\gamma^{\prime} \in \mathcal{T}_{\varphi^{\prime}}$ has edge $I$ of type $i$ and edge $J$ of type $j$, so that (min $J$ $\min I)+\Sigma=w_{j i}^{\prime}$, then $\bar{\rho}\left(\gamma^{\prime}\right)$ has corresponding edges of type $i_{1}$ and $j_{1}$ with initial vertices differing by $P(\min J)-P(\min I)$. That is, $w_{j_{1} i_{1}}=F_{P}\left(w_{j i}^{\prime}\right)$. It follows that if $\tau_{1}$ is translation by $F_{P} u_{i}^{\prime}-u_{i_{1}}$, then

$$
\left(g_{\varphi} \circ \rho\right)_{0}=\tau_{1} \circ F_{\left.P\right|_{V^{\prime}}} \circ\left(g_{\varphi^{\prime}}\right)_{0} .
$$

Similarly, $k$ th coordinates satisfy

$$
\left(g_{\varphi} \circ \rho\right)_{k}=\tau_{1} \circ F_{\left.P\right|_{V^{\prime}}} \circ\left(g_{\varphi^{\prime}}\right)_{k} .
$$

Note that

$$
F_{\left.A\right|_{V}}\left(u_{i_{1}}-F_{\left.P\right|_{V^{\prime}}} u_{i}^{\prime}\right)=F_{\left.P\right|_{V^{\prime}}} u_{i_{1}}-F_{\left.P\right|_{V^{\prime}}} F_{\left.A\right|_{V^{\prime}}} u_{i}^{\prime}=u_{i_{1}}-F_{\left.P\right|_{V^{\prime}}} u_{i}^{\prime}
$$


from the normalization requirement on the $\left\{u_{i}\right\},\left\{u_{i}^{\prime}\right\}$, so translation by this same element in each coordinate in $\lim _{\longleftarrow} F_{\left.A\right|_{V}}$ defines a translation $\tau$ on $\lim _{\longleftarrow} F_{\left.A\right|_{V}}$. We have

$$
g_{\varphi} \circ \bar{\rho}=\tau \circ \hat{F}_{\left.P\right|_{V^{\prime}}} \circ g_{\varphi^{\prime}} .
$$

Thus with $S=\left.P\right|_{V^{\prime}}$ and $T=\left(\left.P\right|_{V^{\prime}}\right)^{-1} \circ\left(\left.A\right|_{V}\right)^{m}$, we have conclusion (3a). The proofs of (2), in case $\varphi=\alpha \circ \beta$ and $\varphi^{\prime}=\beta \circ \alpha$, and (3b) are similar (although more straightforward).

With the notation of Lemma 3 , and with $C_{\varphi}$ and $C_{\varphi^{\prime}}$ the collections of special tilings for $\varphi$ and $\varphi^{\prime}$ resp., note that $\bar{\rho}, \bar{\alpha}$, and $\bar{\beta}$ determine bijections between $C_{\varphi}$ and $C_{\varphi^{\prime}}$, and $\hat{F}_{\left.A\right|_{V}}$ maps $g_{\varphi}\left(C_{\varphi}\right)$ bijectively onto itself. This yields the following consequence of Lemma 3 .

COROLlary 4. If $\varphi$ and $\varphi^{\prime}$ are Pisot substitutions with one a rewriting of the other, or for which there are morphisms $\alpha, \beta$ with $\varphi=\alpha \circ \beta$ and $\varphi^{\prime}=\beta \circ \alpha$, then $\left.A\right|_{V}:(V, \Sigma) \rightarrow(V, \Sigma)$ and $\left.A^{\prime}\right|_{V^{\prime}}:\left(V^{\prime}, \Sigma^{\prime}\right) \rightarrow\left(V^{\prime}, \Sigma^{\prime}\right)$ are shift equivalent, expressed by maps $S:\left(V^{\prime}, \Sigma^{\prime}\right) \rightarrow(V, \Sigma)$ and $T:(V, \Sigma) \rightarrow$ $\left(V^{\prime}, \Sigma^{\prime}\right)$, with

$$
\tau \circ F_{T} \circ \pi_{0} \circ g_{\varphi}\left(C_{\varphi}\right)=\pi_{0} \circ g_{\varphi^{\prime}}\left(C_{\varphi^{\prime}}\right)
$$

and

$$
\tau^{\prime} \circ F_{S} \circ \pi_{0} \circ g_{\varphi^{\prime}}\left(C_{\varphi^{\prime}}\right)=\pi_{0} \circ g_{\varphi}\left(C_{\varphi}\right) .
$$

Let us say that the substitutions $\varphi$ and $\psi$ are in the same rewriting class if there are substitutions $\varphi_{0}=\varphi, \varphi_{1}, \ldots, \varphi_{n}=\psi$ with the property that for each $i=0, \ldots, n-1$, one of $\varphi_{i}$ and $\varphi_{i+1}$ is a rewriting of the other. The proof of the rigidity result in [BSw2] (see Theorem 7 in the next section) establishes the following:

Lemma 5. Suppose that $\varphi$ and $\psi$ are substitutions such that $\mathcal{T}_{\varphi}$ is homeomorphic with $\mathcal{T}_{\psi}$. Then there are $m, n \in \mathbb{N}$ and substitutions $\varphi^{\prime}$ and $\psi^{\prime}$ such that (1) $\varphi^{\prime}$ and $\varphi^{m}$ are in the same rewriting class as are $\psi^{\prime}$ and $\psi^{n}$, and (2) there are morphisms $\alpha$ and $\beta$ such that $\varphi^{\prime}=\alpha \circ \beta$ and $\psi^{\prime}=\beta \circ \alpha$.

Proof. See [BSw2, Theorem 2.1].

To prove Theorem 1, let $\varphi$ and $\psi$ be Pisot of degree $d$, with geometric realizations $g_{\varphi}$ and $g_{\psi}$ of $\mathcal{T}_{\varphi}$ and $\mathcal{T}_{\psi}$. Fix isomorphisms $L_{\varphi}:\left(V_{\varphi}, \Sigma_{\varphi}\right) \rightarrow$ $\left(\mathbb{R}^{d}, \mathbb{Z}^{d}\right)$ and $L_{\psi}:\left(V_{\psi}, \Sigma_{\psi}\right) \rightarrow\left(\mathbb{R}^{d}, \mathbb{Z}^{d}\right)$, and let $\operatorname{Br}(\varphi)=F_{L_{\varphi}} \circ \pi_{0}\left(g_{\varphi}\left(C_{\varphi}\right)\right)$ and $\operatorname{Br}(\psi)=F_{L_{\psi}} \circ \pi_{0}\left(g_{\psi}\left(C_{\psi}\right)\right)$ be the corresponding branch loci. Let $m, n, \varphi^{\prime}$ and $\psi^{\prime}$ be as in Lemma 5 . As $\mathcal{T}_{\varphi}=\mathcal{T}_{\varphi^{m}}$ and $\mathcal{T}_{\psi}=\mathcal{T}_{\psi^{n}}$, we may take $g_{\varphi^{m}}=g_{\varphi}, g_{\psi^{n}}=g_{\psi}, L_{\varphi^{m}}=L_{\varphi}$ and $L_{\psi^{n}}=L_{\psi}$, so that $\operatorname{Br}\left(\varphi^{m}\right)=\operatorname{Br}(\varphi)$ and $\operatorname{Br}\left(\psi^{m}\right)=\operatorname{Br}(\psi)$. Apply Corollary 4 repeatedly to get translations $\eta$ and $\hat{\eta}$ (this last determined by $\eta$ and $\left.L_{\psi}\right)$, and a map $T_{1}:\left(V_{\varphi}, \Sigma_{\varphi}\right) \rightarrow\left(V_{\psi}, \Sigma_{\psi}\right)$ 
for which there are $k, l \in \mathbb{N}$ and a map $S_{1}:\left(V_{\psi}, \Sigma_{\psi}\right) \rightarrow\left(V_{\varphi}, \Sigma_{\varphi}\right)$ such that

$$
S_{1} T_{1}=\left(\left.A_{\varphi}^{m}\right|_{V_{\varphi}}\right)^{k}, \quad T_{1} S_{1}=\left(\left.A_{\psi}^{n}\right|_{V_{\psi}}\right)^{l}
$$

and

$$
\begin{aligned}
\operatorname{Br}(\psi) & =F_{L_{\psi}}\left(\left(g_{\psi^{n}}\left(C_{\psi^{n}}\right)\right)_{0}\right)=F_{L_{\psi}}\left(\eta \circ F_{T_{1}}\left(g_{\varphi^{m}}\left(C_{\varphi^{m}}\right)\right)_{0}\right) \\
& =F_{L_{\psi}}\left(\eta \circ F_{T_{1}}\left(L_{\varphi}^{-1}(\operatorname{Br}(\varphi))\right)=\hat{\eta} \circ F_{L_{\psi} T_{1} L_{\varphi}^{-1}} .\right.
\end{aligned}
$$

If $M_{\varphi}=L_{\varphi} A_{\varphi} L_{\varphi}^{-1}, M_{\psi}=L_{\psi} A_{\psi} L_{\psi}^{-1}, T=L_{\psi} T_{1} L_{\varphi}^{-1}$, and $S=L_{\varphi} S_{1} L_{\psi}^{-1}$ (expressed as matrices in the standard basis on $\mathbb{R}^{d}$ ), $m_{0}=m^{k}$ and $\tau_{0}=\hat{\eta}$, then $\operatorname{Br}(\psi)=\tau_{0} \circ F_{T}(\operatorname{Br}(\varphi))$ with $S T=\left(M_{\varphi}\right)^{m_{0}}$. Similarly, if $m_{1}=n^{l}$, there is a translation $\tau_{1}$ with $\operatorname{Br}(\varphi)=\tau_{1} \circ F_{S}(\operatorname{Br}(\psi))$ and $T S=\left(M_{\psi}\right)^{m_{1}}$.

5. The Pisot part of the augmented dimension group. In this section we show that, for $\varphi$ Pisot of degree $\mathrm{d}$, the relative cohomology group over the integers $H^{1}\left(\mathbb{T}^{d}, \operatorname{Br}(\varphi)\right)$ has a natural order that makes it into a dimension group, which we denote by $\left(\operatorname{PDG}(\varphi), \operatorname{PDG}(\varphi)^{+}\right)$. With an application of Theorem 1 we will demonstrate that $\left(\operatorname{PDG}(\varphi), \operatorname{PDG}(\varphi)^{+}\right)$is a topological invariant of the tiling space $\mathcal{T}_{\varphi}$, and we will see this dimension group is an ordered subgroup of the augmented cohomology group of the tiling space defined in $[\mathrm{BSm}]$. It follows that $\left(\operatorname{PDG}(\varphi), \operatorname{PDG}(\varphi)^{+}\right)$is closely related to the ordered $K_{0}$-group of the Matsumoto algebra of the substitutive system associated with $\varphi$. In fact, had we restricted to only forward asymptotic special tilings in the definition of $\operatorname{Br}(\varphi)$, the resulting $\left(\operatorname{PDG}(\varphi), \operatorname{PDG}(\varphi)^{+}\right)$ would be, essentially, the subgroup of $K_{0}$ corresponding to the restriction of the abelianization of $\varphi$ to the rational invariant subspace spanned by the conjugates of the Perron-Frobenius eigenvector (that is, the "Pisot part" of the abelianization of $\varphi$ ).

For a Pisot substitution $\varphi$ of degree $d$, let $L$ and $M$ be as in the definition of $\operatorname{Br}(\varphi)$. The induced $F_{M}: H^{1}\left(\mathbb{T}^{d} ; \mathbb{R}\right) \emptyset$ is then a vector space isomorphism with simple eigenvalue $\lambda=\lambda_{\varphi}$ and a $(d-1)$-dimensional invariant subspace complementary to the eigenspace of $\lambda$. This $(d-1)$-dimensional space splits $H^{1}\left(\mathbb{T}^{d} ; \mathbb{R}\right)$ into two closed invariant half-spaces, one of which contains the cocycle dual to $\left(F_{L}\right)_{*}([\omega])$, where $[\omega]$ is the 1-cycle generated by the positive eigenvector $\omega$ of $A=A_{\varphi}$. This half-space is called the positive cone in $H^{1}\left(\mathbb{T}^{d} ; \mathbb{R}\right)$ and is denoted by $H^{1}\left(\mathbb{T}^{d} ; \mathbb{R}\right)^{+}$. The inclusion $\imath: \mathbb{Z} \rightarrow \mathbb{R}$ induces $\imath^{*}: H^{1}\left(\mathbb{T}^{d}\right) \rightarrow H^{1}\left(\mathbb{T}^{d} ; \mathbb{R}\right.$ ) (an unspecified coefficient ring is understood to be $\mathbb{Z}$ ), and we define the positive cone in $H^{1}\left(\mathbb{T}^{d}\right)$ to be $H^{1}\left(\mathbb{T}^{d}\right)^{+}:=\left(\imath^{*}\right)^{-1}\left(H^{1}(\mathbb{T} ; \mathbb{R})^{+}\right)$. Likewise, the positive cone in $H^{1}\left(\mathbb{T}^{d}, \operatorname{Br}(\varphi)\right)$ is

$$
H^{1}\left(\mathbb{T}^{d}, \operatorname{Br}(\varphi)\right)^{+}:=\left(\jmath^{*}\right)^{-1}\left(H^{1}\left(\mathbb{T}^{d}\right)^{+}\right),
$$


with $\jmath^{*}: H^{1}\left(\mathbb{T}^{d}, \operatorname{Br}(\varphi)\right) \rightarrow H^{1}\left(\mathbb{T}^{d}\right)$ the natural homomorphism. We have

$$
\begin{aligned}
F_{M}^{*}:\left(H^{1}\left(\mathbb{T}^{d}, \operatorname{Br}(\varphi)\right), H^{1}\left(\mathbb{T}^{d}, \operatorname{Br}(\varphi)\right)^{+}\right) & \\
& \rightarrow\left(H^{1}\left(\mathbb{T}^{d}, \operatorname{Br}(\varphi)\right), H^{1}\left(\mathbb{T}^{d}, \operatorname{Br}(\varphi)\right)^{+}\right),
\end{aligned}
$$

and we define $\operatorname{PDG}(\varphi):=\lim _{(}\left(F_{B}^{*}: H^{1}\left(\mathbb{T}^{d}, \operatorname{Br}(\varphi)\right) \rightarrow H^{1}\left(\mathbb{T}^{d}, \operatorname{Br}(\varphi)\right)\right)$, with positive cone

$$
\operatorname{PDG}(\varphi)^{+}:=\left\{[(k, g)] \in \operatorname{PDG}(\varphi): g \in H^{1}\left(\mathbb{T}^{d}, \operatorname{Br}(\varphi)\right)^{+}\right\} .
$$

The shift isomorphism on $\left(\operatorname{PDG}(\varphi), \operatorname{PDG}(\varphi)^{+}\right)$is the ordered isomorphism given by $[(k, g)] \mapsto\left[\left(k, F_{M}^{*}(g)\right)\right]$.

A homeomorphism of tiling spaces, $f: \mathcal{T}_{\varphi} \rightarrow \mathcal{T}_{\psi}$, is orientation preserving (resp. reversing) provided it takes the positive flow direction in $\mathcal{T}_{\varphi}$ to the positive (resp. negative) flow direction in $\mathcal{T}_{\psi}$; that is, the function $s: \mathcal{T}_{\varphi} \times$ $\mathbb{R}^{+} \rightarrow \mathbb{R}$ defined by $f(\gamma-t)=f(\gamma)-s(\gamma, t)$ is positive (negative).

THEOREM 6. If $\varphi$ and $\psi$ are Pisot substitutions, and the tiling spaces $\mathcal{T}_{\varphi}$ and $\mathcal{T}_{\psi}$ are orientation preserving (reversing) homeomorphic, then there is an ordered isomorphism between the pairs $\left(\operatorname{PDG}(\varphi), \operatorname{PDG}(\varphi)^{+}\right)$and $\left(\operatorname{PDG}(\psi), \operatorname{PDG}(\psi)^{+}\right)$(resp. $\left.\left(\operatorname{PDG}(\psi),-\operatorname{PDG}(\psi)^{+}\right)\right)$that conjugates some positive powers of the shift isomorphisms.

Proof. Let $T, S, \tau_{0}, \tau_{1}, m_{0}$, and $m_{1}$ be as in Theorem 1 . The commuting diagram

$$
H^{1}\left(\mathbb{T}^{d}, \operatorname{Br}(\varphi)\right) \stackrel{F_{M_{\varphi}^{*} m_{0}}^{\longrightarrow}}{\longrightarrow} H^{1}\left(\mathbb{T}^{d}, \operatorname{Br}(\varphi)\right)
$$

induces an isomorphism $[(k, g)] \rightarrow\left[\left(k,\left(\tau_{1} \circ F_{S}\right)^{*}(g)\right)\right]$ that conjugates the $m_{1}$ th power of the shift on $\operatorname{PDG}(\psi)$ with the $m_{0}$ th power of the shift on $\operatorname{PDG}(\varphi)$. Since the order is dynamically defined, this conjugacy alone guarantees that order is either preserved or reversed. If $\mathcal{T}_{\varphi}$ and $\mathcal{T}_{\psi}$ are orientation preserving homeomorphic, then $S$ and $T$ take $L_{\psi}\left(\omega_{\psi}\right)$ and $L_{\varphi}\left(\omega_{\varphi}\right)$ to positive multiples of $L_{\varphi}\left(\omega_{\varphi}\right)$ and $L_{\psi}\left(\omega_{\psi}\right)$, resp. (here, $L_{\varphi}$ and $L_{\psi}$ denote the $L$ in the definition of $\operatorname{Br}(\varphi)$ and $\operatorname{Br}(\psi)$, and $\omega_{\varphi}, \omega_{\psi}$ are positive eigenvectors of $A_{\varphi}, A_{\psi}$, resp.). Thus $\left(\tau_{1} \circ F_{S}\right)^{*}\left(H^{1}\left(\mathbb{T}^{d}, \operatorname{Br}(\varphi)\right)^{+}\right)=H^{1}\left(\mathbb{T}^{d}, \operatorname{Br}(\psi)\right)^{+}$.

If $\mathcal{T}_{\varphi}$ and $\mathcal{T}_{\psi}$ are orientation reversing homeomorphic, then $\mathcal{T}_{\varphi}$ and $\mathcal{T}_{\bar{\psi}}$ are orientation preserving homeomorphic, $\bar{\psi}$ being the reverse of $\psi$ (if $\psi(i)=$ $i_{1} \cdots i_{k}$ then $\left.\bar{\psi}(i)=i_{k} \cdots i_{1}\right)$. The above applied to $\left(\operatorname{PDG}(\varphi), \operatorname{PDG}(\varphi)^{+}\right)$ 
and $\left(\operatorname{PDG}(\psi), \operatorname{PDG}(\psi)^{+}\right)$together with the isomorphism

$$
\left(\operatorname{PDG}(\bar{\psi}), \operatorname{PDG}(\bar{\psi})^{+}\right) \rightarrow\left(\operatorname{PDG}(\psi),-\operatorname{PDG}(\psi)^{+}\right)
$$

induced by $F_{-I d}^{*}$ yields the conclusion of the theorem in the orientation reversing case.

Example 6. Consider

$$
\varphi:\left\{\begin{array}{l}
a \rightarrow a b a b b a \\
b \rightarrow a a b b b a
\end{array} \text { and } \quad \psi:\left\{\begin{array}{l}
a \rightarrow a^{47} a b^{18} b b^{29} \\
b \rightarrow a^{47} b b^{18} a b^{29} .
\end{array}\right.\right.
$$

One computes

$$
\begin{aligned}
& \left(\operatorname{PDG}(\varphi), \operatorname{PDG}(\varphi)^{+}\right) \cong\left(\operatorname{DG}\left(\begin{array}{ll}
6 & 0 \\
1 & 1
\end{array}\right), \operatorname{DG}\left(\begin{array}{ll}
6 & 0 \\
1 & 1
\end{array}\right)^{+}\right) \\
& \left(\operatorname{PDG}(\psi), \operatorname{PDG}(\psi)^{+}\right) \cong\left(\operatorname{DG}\left(\begin{array}{ll}
96 & 0 \\
19 & 1
\end{array}\right), \operatorname{DG}\left(\begin{array}{ll}
96 & 0 \\
19 & 1
\end{array}\right)^{+}\right) .
\end{aligned}
$$

These groups are order isomorphic but, because no power of 96 is a power of 6 , the shift isomorphisms do not have conjugate powers. By Theorem 6 , $\mathcal{T}_{\varphi}$ and $\mathcal{T}_{\psi}$ are not homeomorphic.

We now relate the pair $\left(\operatorname{PDG}(\varphi), \operatorname{PDG}(\varphi)^{+}\right)$to the ordered augmented cohomology of the tiling space $\mathcal{T}_{\varphi}$. We begin with a description of the augmented tiling space (for more detail, and a precise description as an inverse limit, see $[\mathrm{BSm}])$.

Let $\varphi$ be any primitive aperiodic substitution with tiling space $\mathcal{T}_{\varphi}$. Let $\left\{\gamma_{1}^{f}, \ldots, \gamma_{n_{f}}^{f}\right\}$ be a collection of forward asymptotic special tilings, exactly one chosen from each forward asymptotic equivalence class, and $\left\{\gamma_{1}^{b}, \ldots, \gamma_{n_{b}}^{b}\right\}$ a collection of backward asymptotic special tilings, one from each backward asymptotic equivalence class. Let $R_{j}^{f}, j=1, \ldots, n_{f}$, and $R_{i}^{b}, i=1, \ldots, n_{b}$, be the rays

$$
R_{j}^{f}:=\left\{\{j\} \times\left\{\gamma_{j}^{f}-t\right\}: t \geq 0\right\} \quad \text { and } \quad R_{i}^{b}:=\left\{\{i\} \times\left\{\gamma_{i}^{b}-t\right\}: t \geq 0\right\} .
$$

The augmented tiling space $\widetilde{\mathcal{T}}_{\varphi}$ is defined to be the union

$$
\widetilde{\mathcal{T}}_{\varphi}:=\mathcal{T}_{\varphi} \cup\left(\bigcup_{j=1}^{n_{f}} R_{j}^{f} \cup \bigcup_{i=1}^{n_{b}} R_{i}^{b}\right) /\left\{\{j\} \times\left\{\gamma_{j}^{f}\right\},\{i\} \times\left\{\gamma_{i}^{b}\right\}\right\},
$$

in which all of the endpoints of the rays have been identified to a single branch point. The metric on $\mathcal{T}_{\varphi}$ is extended to $\widetilde{\mathcal{T}}_{\varphi}$ in such a way that

$$
d\left(\gamma_{j}^{f}-t,\{j\} \times\left\{\gamma_{j}^{f}-t\right\}\right)=\frac{1}{1+t}=d\left(\gamma_{i}^{b}+t,\{i\} \times\left\{\gamma_{i}^{b}+t\right\}\right)
$$

for $t \geq 0$, making the ray $R_{j}^{f}$ asymptotic to the forward orbit of $\gamma_{j}^{f}$, and the ray $R_{i}^{b}$ asymptotic to the backward orbit of $\gamma_{i}^{b}$. 
The homeomorphism $\Phi$ extends to $\widetilde{\Phi}: \widetilde{\mathcal{T}}_{\varphi} \oslash$ with

$$
\widetilde{\Phi}\left(\{j\} \times\left\{\gamma_{j}^{f}-t\right\}\right)=\left\{j^{\prime}\right\} \times\left\{\gamma_{j^{\prime}}^{f}-\lambda t\right\},
$$

where $j^{\prime}$ is such that $\Phi\left(\gamma_{j}^{f}\right)$ is forward asymptotic to $\gamma_{j^{\prime}}^{f}$, and similarly for the rays $R_{i}^{b}$. We order $H^{1}\left(\widetilde{\mathcal{T}}_{\varphi}\right)$ in such a way that $\widetilde{\Phi}^{*}:\left(H^{1}\left(\widetilde{\mathcal{T}}_{\varphi}\right), H^{1}\left(\widetilde{\mathcal{T}}_{\varphi}\right)^{+}\right) \emptyset$ is an order isomorphism. First note that $\Phi^{*}: H^{1}\left(\mathcal{T}_{\varphi} ; \mathbb{R}\right) \emptyset$ is a vector space isomorphism with simple eigenvalue $\lambda=\lambda_{\varphi}$ and codimension one invariant subspace complementary to the eigenspace of $\lambda$. This codimension one space splits $H^{1}\left(\mathcal{T}_{\varphi}\right)$ into two closed half-spaces, one of which corresponds to the positive direction of the flow $\left({ }^{4}\right)$; call that space $H^{1}\left(\mathcal{T}_{\varphi} ; \mathbb{R}\right)^{+}$. Let $H^{1}\left(\widetilde{\mathcal{T}}_{\varphi}\right)^{+}=$ $\left(\imath_{*} \jmath^{*}\right)^{-1}\left(H^{1}\left(\mathcal{T}_{\varphi} ; \mathbb{R}\right)^{+}\right)$, where $\imath_{*}: H^{1}\left(\mathcal{T}_{\varphi}\right) \rightarrow H^{1}\left(\mathcal{T}_{\varphi} ; \mathbb{R}\right)$ is induced by $\imath: \mathbb{Z}$ $\rightarrow \mathbb{R}$ and $\jmath^{*}: H^{1}\left(\widetilde{\mathcal{T}}_{\varphi}\right) \rightarrow H^{1}\left(\mathcal{T}_{\varphi}\right)$ is induced by the inclusion $j: \mathcal{T}_{\varphi} \rightarrow \widetilde{\mathcal{T}}_{\varphi}$. The pair $\left(H^{1}\left(\widetilde{\mathcal{T}}_{\varphi}\right), H^{1}\left(\widetilde{\mathcal{T}}_{\varphi}\right)^{+}\right)$is the ordered augmented cohomology of $\mathcal{T}_{\varphi}$. Call the isomorphism $\widetilde{\Phi}^{*}:\left(H^{1}\left(\widetilde{\mathcal{T}}_{\varphi}\right), H^{1}\left(\widetilde{\mathcal{T}}_{\varphi}\right)^{+}\right) \emptyset$ the shift isomorphism.

The rigidity result of $[\mathrm{BSw} 2]$ is

THEOREM 7. If $f: \mathcal{T}_{\varphi} \rightarrow \mathcal{T}_{\psi}$ is a homeomorphism of substitution tiling spaces, then there are $m, n \in \mathbb{N}$ and $h: \mathcal{T}_{\varphi} \rightarrow \mathcal{T}_{\psi}$ isotopic to $f$ such that $h \circ \Phi^{m}=\Psi^{n} \circ h$.

COROLlaRY 8. If $\mathcal{T}_{\varphi}$ and $\mathcal{T}_{\psi}$ are orientation preserving (reversing) homeomorphic, then the ordered cohomologies $\left(H^{1}\left(\widetilde{\mathcal{T}}_{\varphi}\right), H^{1}\left(\widetilde{\mathcal{T}}_{\varphi}\right)^{+}\right)$and $\left(H^{1}\left(\widetilde{\mathcal{T}}_{\psi}\right), H^{1}\left(\widetilde{\mathcal{T}}_{\psi}\right)^{+}\right)\left(\right.$resp.,$\left.\left(H^{1}\left(\widetilde{\mathcal{T}}_{\psi}\right),-H^{1}\left(\widetilde{\mathcal{T}}_{\psi}\right)^{+}\right)\right)$are isomorphic by an isomorphism that conjugates some positive powers of the shift isomorphisms.

Proof. The homeomorphism $h$ of Theorem 7 extends to a homeomorphism $\widetilde{h}: \widetilde{\mathcal{T}}_{\varphi} \rightarrow \widetilde{\mathcal{T}}_{\psi}$ that conjugates $\widetilde{\Phi}^{m}$ with $\widetilde{\Psi}^{n}$.

The corollary - without the conjugacy in the conclusion-appears in [BSm]. Also, in that paper, a nonnegative integer matrix $\widetilde{A}^{t}$ is constructed with ordered dimension group $\left(\operatorname{DG}\left(\widetilde{A}^{t}\right), \operatorname{DG}\left(\widetilde{A}^{t}\right)^{+}\right)$isomorphic to $\left(H^{1}\left(\widetilde{\mathcal{T}}_{\varphi}\right)\right.$, $\left.H^{1}\left(\widetilde{\mathcal{T}}_{\varphi}\right)^{+}\right)$.

Viewing $\left(\operatorname{PDG}(\varphi), \operatorname{PDG}(\varphi)^{+}\right)$as the Pisot part of the augmented cohomology is justified by our final theorem. A formula for the Pisot part as the dimension group of an integer matrix will arise in the proof.

TheOREM 9. If $\varphi$ is a Pisot substitution, then there is an ordered embedding of $\left(\operatorname{PDG}(\varphi), \operatorname{PDG}(\varphi)^{+}\right)$into $\left(H^{1}\left(\widetilde{\mathcal{T}}_{\varphi}\right), H^{1}\left(\widetilde{\mathcal{T}}_{\varphi}\right)^{+}\right)$that commutes with a positive power of the shift isomorphisms.

$\left({ }^{4}\right)$ Specifically, there is an orientation preserving map of $\mathcal{T}_{\varphi}$ onto the circle $\mathbb{T}^{1}$. Let $H^{1}\left(\mathcal{T}_{\varphi} ; \mathbb{R}\right)^{+}$contain the positive half-line in $H^{1}\left(\mathcal{T}^{1} ; \mathbb{R}\right)$ pulled back to $H^{1}\left(\mathcal{T}_{\varphi} ; \mathbb{R}\right)$. 
Example 7 . If $\varphi$ is the Thue-Morse substitution $(1 \rightarrow 12,2 \rightarrow 21)$, then

$$
\left(\operatorname{PDG}(\varphi), \operatorname{PDG}(\varphi)^{+}\right)=\left(\mathbb{Z}\left[\frac{1}{2}\right],\left\{x \in \mathbb{Z}\left[\frac{1}{2}\right]: x \geq 0\right\}\right)
$$

with shift isomorphism $x \mapsto 2 x$, and

$$
\left(H^{1}\left(\widetilde{\mathcal{T}}_{\varphi}\right), H^{1}\left(\widetilde{\mathcal{T}}_{\varphi}\right)^{+}\right)=\left(\mathbb{Z}\left[\frac{1}{2}\right] \oplus \mathbb{Z}^{4},\left\{(x, y) \in \mathbb{Z}\left[\frac{1}{2}\right] \oplus \mathbb{Z}^{4}: x \geq 0\right\}\right)
$$

with shift isomorphism $(x, y) \mapsto(2 x, y)$.

Proof of Theorem 9. Let $\varphi$ be a Pisot substitution. To simplify, we pass to a prepared substitution (see [BSm]). We may suppose all special asymptotic tilings in $\mathcal{T}_{\varphi}$ are fixed by $\varphi^{n}$, for some $n \in \mathbb{N}$, and $\varphi^{n}$ has a fixed bi-infinite word $\cdots b . a \cdots$. So we have $\varphi^{n}(a)=a \cdots, \varphi^{n}(b)=\cdots b$ and $b a$ occurs in $\varphi^{k}(a)$ for some $k \in \mathbb{N}$.

We rewrite $\varphi^{n}$ with stopping rule $b$ and starting rule $a$ as follows. Let $\mathcal{U}=\left\{u_{1}, \ldots, u_{q}\right\}$ be the finite collection of words with the properties:

(i) each $u_{i}$ occurs as a factor of the infinite word $a \varphi^{n}(a) \varphi^{2 n}(a) \cdots$;

(ii) each $u_{i}$ has the form $a \cdots b$;

(iii) $b a$ is not a factor of any $u_{i}, i=1, \ldots, q$.

Then each word $\varphi^{n}\left(u_{i}\right)$ factors uniquely in the form $u_{i_{1}} \cdots u_{i_{p}}$. From this, define $\psi$ by $\psi(i)=i_{1} \cdots i_{p}, i=1, \ldots, q$. The substitution $\psi$ is primitive and aperiodic Pisot $\left(\lambda_{\psi}=\lambda_{\varphi}^{n}\right)$ and strictly proper: there are $r, s \in$ $\{1, \ldots, q\}$ such that $\psi(i)=r \cdots s$ for $i=1, \ldots, q$. Also, the special asymptotic tilings in $\mathcal{T}_{\psi}$ are all fixed by $\Psi$. Then $\mathcal{T}_{\varphi^{n}}=\mathcal{T}_{\varphi}$ and $\mathcal{T}_{\psi}$ are orientation preserving homeomorphic (this is a special case of Lemma 3 ) by a homeomorphism that conjugates $\Phi^{n}$ with $\Psi$. Hence, $\left(H^{1}\left(\widetilde{\mathcal{T}}_{\varphi}\right), H^{1}\left(\widetilde{\mathcal{T}}_{\varphi}\right)^{+}\right)$and $\left(H^{1}\left(\widetilde{\mathcal{T}}_{\psi}\right), H^{1}\left(\widetilde{\mathcal{T}}_{\psi}\right)^{+}\right)$are isomorphic by an isomorphism that conjugates $\left(\Phi^{n}\right)^{*}$ with $\Psi^{*}($ Corollary 8$)$, and $\left(\operatorname{PDG}(\varphi), \operatorname{PDG}(\varphi)^{+}\right)=\left(\operatorname{PDG}\left(\varphi^{n}\right), \operatorname{PDG}\left(\varphi^{n}\right)^{+}\right)$ is isomorphic to $\left(\operatorname{PDG}(\psi), \operatorname{PDG}(\psi)^{+}\right)$(Theorem 6$)$. The isomorphism conjugates the $(\ln )$ th power of the shift on $\left(\operatorname{PDG}(\varphi), \operatorname{PDG}(\varphi)^{+}\right)$to the $l$ th power of the shift on $\left(\operatorname{PDG}(\psi), \operatorname{PDG}(\psi)^{+}\right)$for some $l \in \mathbb{N}$. Thus once we prove the theorem for such substitutions $\psi$, we have proved it for all Pisot substitutions.

Let $n_{f}$ denote the number of equivalence classes of forward asymptotic special tilings in $\mathcal{T}_{\psi}$, and $n_{b}$ the number of special backward classes. As in [BSm], we may select special asymptotic tilings $\gamma_{j}^{f}, j=1, \ldots, n_{f}$, one from each forward class, and $\gamma_{i}^{b}, i=1, \ldots, n_{b}$, one from each backward class, with the properties

(i) if $J$ is an edge of $\gamma_{j}^{f}$ (resp., $I$ an edge of $\gamma_{i}^{b}$ ) that meets $\mathbb{E}^{s}$, then $J$ (resp., $I$ ) meets $\mathbb{E}^{s}$ in its interior; 
(ii) if $k$ is the type of $J$ (resp., of $I$ ), then $\psi(k)=p_{j}^{f} k s_{j}^{f}$ (resp., $\psi(k)=$ $\left.p_{i}^{b} k s_{i}^{b}\right)$ with $p_{j}^{f}$ and $s_{j}^{f}$ (resp., $p_{i}^{b}$ and $s_{i}^{b}$ ) nonempty and

$$
\begin{aligned}
w_{j}^{f}:=\cdots \psi^{2}\left(p_{j}^{f}\right) \psi\left(p_{j}^{f}\right) p_{j}^{f} \psi\left(s_{j}^{f}\right) \psi^{2}\left(s_{j}^{f}\right) \cdots & \\
& \quad\left(\text { resp., } w_{i}^{b}:=\cdots \psi\left(p_{i}^{b}\right) p_{i}^{b} \psi\left(s_{i}^{b}\right) \cdots\right)
\end{aligned}
$$

is the bi-infinite word corresponding to $\gamma_{j}^{f}$ (resp., $\gamma_{i}^{b}$ ).

In particular, and this will be important later,

$$
A(\min I)+\operatorname{pr}_{V} \llbracket p_{i}^{b} \rrbracket=\min I \quad \text { and } \quad A(\max J)-\operatorname{pr}_{V} \llbracket p_{j}^{f} \rrbracket=\max J,
$$

where $\llbracket u \rrbracket$ denotes the abelianization of the word $u$.

Let $E$ denote the $n \times\left(n_{f}+n_{b}-1\right)$ matrix with $i j$ th entry $E_{i j}= \begin{cases}\text { number of occurrences of } i \text { in } p_{1}^{b} s_{j}^{f} & \text { if } 1 \leq j \leq n_{f}, \\ \text { number of occurrences of } i \text { in } p_{j-n_{f}+1}^{b} s_{1}^{f} & \text { if } n_{f}<j \leq n_{f}+n_{b}-1 .\end{cases}$

Let $A=A_{\psi}$ be the abelianization of $\psi$, and let $I$ be the $\left(n_{f}+n_{b}-1\right) \times$ $\left(n_{f}+n_{b}-1\right)$ identity matrix. The augmented matrix for $\psi$ is

$$
\widetilde{A}=\left(\begin{array}{cc}
A & E \\
O & I
\end{array}\right)
$$

of size $\widetilde{n}=n+n_{f}+n_{b}-1$. The augmented dimension group for $\psi$ is the pair

$$
\left(\operatorname{DG}\left(\widetilde{A}^{t}\right), \operatorname{DG}\left(\widetilde{A}^{t}\right)^{+}\right), \quad \text { where } \quad \operatorname{DG}\left(\widetilde{A}^{t}\right):=\lim _{\longrightarrow} \widetilde{A}^{t}: \mathbb{Z}^{\widetilde{n}} \emptyset
$$

with $\operatorname{DG}\left(\widetilde{A}^{t}\right)^{+}$determined (dynamicallly) as before:

Since $\lambda_{\psi}$ is simple, $\widetilde{A}^{t}$ has a codimension one invariant subspace $W$ (in $\mathbb{R}^{\widetilde{n}}$ ) complementary to the eigenspace of $\lambda_{\psi}$. Let

$\left(\mathbb{Z}^{\widetilde{n}}\right)^{+}:=\left\{x \in \mathbb{Z}^{\widetilde{n}}: x\right.$ is in the half-space determined by $W$ containing the nonnegative eigenvector corresponding to $\left.\lambda_{\psi}\right\}$. Then $\operatorname{DG}\left(\widetilde{A}^{t}\right)^{+}:=\left\{[k, g] \in \operatorname{DG}\left(\widetilde{A}^{t}\right): g \in\left(\mathbb{Z}^{\widetilde{n}}\right)^{+}\right\}$.

We know from $[\mathrm{BSm}]$ that $\left(\operatorname{DG}\left(\widetilde{A}^{t}\right), \operatorname{DG}\left(\widetilde{A}^{t}\right)^{+}\right)$is isomorphic to $\left(H^{1}\left(\widetilde{\mathcal{T}}_{\psi}\right)\right.$, $\left.H^{1}\left(\widetilde{\mathcal{T}}_{\psi}\right)^{+}\right)$via an isomorphism that conjugates the shifts. Our remaining task, then, is to embed $\left(\operatorname{PDG}(\psi), \operatorname{PDG}(\psi)^{+}\right)$into $\left(\operatorname{DG}\left(\widetilde{A}^{t}\right), \operatorname{DG}\left(\widetilde{A}^{t}\right)^{+}\right)$.

Besides enabling us to describe its augmented cohomology as a dimension group, another advantage of a prepared substitution (like $\psi$ ) is that its return lattice $\Sigma$ is the same as $\Gamma=\operatorname{pr}_{V}\left(\mathbb{Z}^{n}\right)$, making formulas for geometric realization simpler. To show these lattices agree, let $\gamma \in \mathcal{T}_{\psi}$, and let $J$ be an edge of type $j$ in $\gamma$ followed by the edge $I$ of type $i$. Since $\psi$ is strictly proper, $\Psi(J)$ begins with an edge of the same type, say $r$, as does $\Psi(I)$. 
Thus

$$
\begin{aligned}
\min \Psi(I)-\min \Psi(J) & =\max \Psi(J)-\min \Psi(J)=\operatorname{pr}_{V}\left(A e_{j}\right) \\
& =\left(\left.A\right|_{V}\right) \operatorname{pr}_{V} e_{j}=\left(\left.A\right|_{V}\right) v_{j} \in \Theta(j) .
\end{aligned}
$$

So, $v_{j} \in \Sigma$ for all $j$, and $\Sigma=\Gamma$.

We may suppose, without loss of generality, that $\left\{v_{1}, \ldots, v_{d}\right\}$ is a basis for $\Sigma=\Gamma$, taken from the basis $\left\{v_{1}, \ldots, v_{n}\right\}$ for $\mathbb{R}^{n}$. Let $\overline{\operatorname{Br}}(\psi)=\pi_{0} g_{\psi}\left(\mathcal{C}_{\psi}\right)$ be the branch locus in $V / \Sigma$ (so $F_{L_{\psi}}(\overline{\operatorname{Br}}(\psi))=\operatorname{Br}(\psi)$ ). If $\gamma$ and $\gamma^{\prime}$ are asymptotic in $\mathcal{T}_{\psi}$, then $\pi_{0} g_{\psi}(\gamma)$ and $\pi_{0} g_{\psi}\left(\gamma^{\prime}\right)$ are asymptotic in $V / \Sigma$ under the Kronecker flow; that is, $\pi_{0} g_{\psi}(\gamma)=\pi_{0} g_{\psi}\left(\gamma^{\prime}\right)$. It follows that

$$
\overline{\operatorname{Br}}(\psi)=\left\{\pi_{0} g_{\psi}\left(\gamma_{1}^{f}\right), \ldots, \pi_{0} g_{\psi}\left(\gamma_{n_{f}}^{f}\right)\right\} \cup\left\{\pi_{0} g_{\psi}\left(\gamma_{1}^{b}\right), \ldots, \pi_{0} g_{\psi}\left(\gamma_{n_{b}}^{b}\right)\right\} .
$$

If $I$ is the unique edge of $\gamma_{i}^{b}$ that meets $\mathbb{E}^{s}$, let $x_{i}^{b}:=\min I$; and if $J$ is the unique edge of $\gamma_{j}^{f}$ that meets $\mathbb{E}^{s}$, let $x_{j}^{f}:=\max J$.

Since $\Sigma=\Gamma$, we may take the $u_{i}=0$ in the definition of geometric realization $g_{\psi}$. Thus $\pi_{0} g_{\psi}\left(\gamma_{j}^{f}\right)=x_{j}^{f}+\Sigma=: \bar{x}_{j}^{f}$ and $\pi_{0} g_{\psi}\left(\gamma_{i}^{b}\right)=x_{i}^{b}+\Sigma=: \bar{x}_{i}^{b}$.

If $\# \overline{\operatorname{Br}}(\psi)=1$, then

$$
\left(\operatorname{PDG}(\psi), \operatorname{PDG}(\psi)^{+}\right) \cong\left(\left.\lim _{\longrightarrow} A\right|_{V},\left(\left.\lim _{\longrightarrow} A\right|_{V}\right)^{+}\right),
$$

which is easily embedded in (DG $\left.(\psi), \operatorname{DG}(\psi)^{+}\right)$.

Thus we can assume $\# \operatorname{Br}(\psi)=m_{f}+m_{b}$ with $m_{f}>0$ and $m_{b}>0$, and after reindexing,

$$
\overline{\operatorname{Br}}(\psi)=\left\{\bar{x}_{i}^{b}: i=1, \ldots, m_{b}\right\} \cup\left\{\bar{x}_{j}^{f}: j=1, \ldots, m_{f}\right\} .
$$

Recall that $\sigma_{i}:=\left\{t v_{i}: t \in[0,1]\right\}$ is the oriented segment. The homology classes $\left[\bar{\sigma}_{i}\right]$ of the oriented cycles $\bar{\sigma}_{i}:=\sigma_{i}+\Sigma, i=1, \ldots, d$, constitute a basis for $H_{1}(V / \Sigma)$.

For each $i=1, \ldots, n_{b}$, let $\alpha_{i}$ denote the oriented line segment in $V$ from $x_{i}^{b}$ to 0 ; for each $j=1, \ldots, n_{f}$, let $\beta_{j}$ be the directed line segment from 0 to $x_{j}^{f}$; and let $\bar{\alpha}_{i}:=\alpha_{i}+\Sigma, \bar{\beta}_{j}:=\beta_{j}+\Sigma$. Then

$$
\left\{\left[\bar{\sigma}_{1}\right], \ldots,\left[\bar{\sigma}_{\alpha}\right],\left[\bar{\alpha}_{1} \bar{\beta}_{1}\right],\left[\bar{\alpha}_{1} \bar{\beta}_{2}\right], \ldots,\left[\bar{\alpha}_{1} \bar{\beta}_{m_{f}}\right],\left[\bar{\alpha}_{2} \bar{\beta}_{1}\right],\left[\bar{\alpha}_{3} \bar{\beta}_{1}\right], \ldots,\left[\bar{\alpha}_{m_{b}} \bar{\beta}_{1}\right]\right\}
$$

is a basis for $H_{1}(V / \Sigma, \overline{\operatorname{Br}}(\psi))$.

Let $\mathcal{L}: \mathbb{Z}^{\widetilde{n}} \rightarrow \mathbb{Z}^{\widetilde{n}}$ (recall $\widetilde{n}=n+n_{f}+n_{b}-1$ ) denote the homomorphism represented by $\widetilde{A}$ in the standard basis $\mathcal{B}=\left\{e_{1}, \ldots, e_{\tilde{n}}\right\}$. For convenience, relabel the basis elements as follows:

$$
e_{i j}= \begin{cases}e_{i} & \text { for } j=0 \text { and } i=1, \ldots, n, \\ e_{n+j} & \text { for } i=1 \text { and } j=1, \ldots, n_{f}, \\ e_{n+n_{f}+i-1} & \text { for } j=1 \text { and } i=2, \ldots, n_{b} .\end{cases}
$$


We define a homomorphism

$$
P: \mathbb{Z}^{\widetilde{n}} \rightarrow H_{1}(V / \Sigma, \overline{\operatorname{Br}}(\psi))
$$

on the basis $\mathcal{B}$ as follows:

$$
\begin{aligned}
& P\left(e_{i 0}\right):=\sum_{j=1}^{d} r_{i j}\left[\bar{\sigma}_{j}\right], \quad \text { provided } \operatorname{pr}_{V}\left(v_{i}\right)=\sum_{j=1}^{d} r_{i j} v_{j}, i=1, \ldots, n ; \\
& P\left(e_{i j}\right):=\left[\bar{\alpha}_{i} \bar{\beta}_{j}\right], \quad i=1, j=1, \ldots, n_{f} \text { and } j=1, i=2, \ldots, n_{b} .
\end{aligned}
$$

Clearly, $P$ is surjective. The relation $P \mathcal{L}=\left(F_{\left.A\right|_{V}}\right)_{*} P$ evidently holds on the basis elements $e_{10}, \ldots, e_{n 0}$, so consider $e_{i j} \in \mathcal{B}$ with $j>0$. Let $p_{i}:=$ $\llbracket p_{i}^{b} \rrbracket \in \mathbb{Z}^{n}, s_{j}:=\llbracket s_{j}^{f} \rrbracket \in \mathbb{Z}^{n}$, and let $\imath: \mathbb{Z}^{n} \rightarrow \mathbb{Z}^{\widetilde{n}}$ be given by $\imath\left(x_{1}, \ldots, x_{n}\right)=$ $\sum_{i=1}^{n} x_{i} e_{i 0}$. Then $\mathcal{L}\left(e_{i j}\right)=\imath\left(p_{i}\right)+\imath\left(s_{j}\right)+e_{i j}$ and

$$
\begin{aligned}
P \mathcal{L}\left(e_{i j}\right) & =P \mathcal{L}\left(p_{i}\right)+P \mathcal{L}\left(s_{j}\right)+P\left(e_{i j}\right) \\
& =\sum_{k=1}^{d} a_{k}\left[\bar{\sigma}_{k}\right]+\sum_{k=1}^{d} b_{k}\left[\bar{\sigma}_{k}\right]+\left[\bar{\alpha}_{i} \bar{\beta}_{j}\right],
\end{aligned}
$$

where $\operatorname{pr}_{V}\left(p_{i}\right)=\sum_{k=1}^{d} a_{k} v_{k}$ and $\operatorname{pr}_{V}\left(s_{j}\right)=\sum_{k=1}^{d} b_{k} v_{k}$.

On the other hand,

$$
\left(F_{\left.A\right|_{V}}\right)_{*} P\left(e_{i j}\right)=\left(F_{\left.A\right|_{V}}\right)_{*}\left(\left[\bar{\alpha}_{i} \bar{\beta}_{j}\right]\right)=\left[\overline{A\left(\alpha_{i} \beta_{j}\right)}\right],
$$

if $A\left(\alpha_{i} \beta_{j}\right)$ denotes the image of the directed curve $\alpha_{i} \beta_{j}$ under the linear map $A: V \rightarrow V$. We claim that $\overline{A\left(\alpha_{i} \beta_{j}\right)}$ is homologous to $\bar{\rho}_{i}+\bar{\alpha}_{i} \bar{\beta}_{j}+\bar{\eta}_{j}$, where $\rho_{i}:=\left\{t \operatorname{pr}_{V}\left(p_{i}\right): 0 \leq t \leq 1\right\}$ and $\eta_{j}:=\left\{t \operatorname{pr}_{V}\left(s_{j}\right): 0 \leq t \leq 1\right\}$ are directed segments. Indeed, this follows from $A\left(-x_{i}^{b}\right)=\operatorname{pr}_{V}\left(p_{i}\right)+\left(-x_{i}^{b}\right)$ and $A x_{j}^{f}=x_{j}^{f}+\operatorname{pr}_{V}\left(s_{j}\right)$ in the cover $V$ of $V / \Sigma$ (see Figure 1). Clearly $\bar{\rho}_{i}$ is homologous to $\sum_{k=1}^{d} a_{k} \bar{\sigma}_{k}$ and $\bar{\eta}_{j}$ is homologous to $\sum_{k=1}^{d} b_{k} \bar{\sigma}_{k}$. Thus $P \mathcal{L}\left(e_{i j}\right)=\left(F_{\left.A\right|_{V}}\right)_{*} P\left(e_{i j}\right)$.

Since $P$ is surjective, the dual

$$
P^{t}: \operatorname{Hom}\left(H_{1}(V / \Sigma, \overline{\operatorname{Br}}(\psi)), \mathbb{Z}\right) \cong H^{1}(V / \Sigma, \overline{\operatorname{Br}}(\psi)) \rightarrow \operatorname{Hom}\left(\mathbb{Z}^{\widetilde{n}}, \mathbb{Z}\right) \cong \mathbb{Z}^{\widetilde{n}}
$$

is injective. As $\mathcal{L}^{t} P^{t}=P^{t}\left(F_{\left.A\right|_{V}}\right)^{*}$, we know $P^{t}$ induces an injection

$$
\widehat{P}^{t}: \lim _{A \mid V} F_{A \mid}=\operatorname{PDG}(\psi) \rightarrow \underline{\lim } \mathcal{L}^{t}=\operatorname{DG}(\psi)
$$

that commutes with the shifts. Since the positive cones are determined dynamically, $\widehat{P}^{t}$ either preserves or reverses order. As $\left[\sigma_{1}\right]^{*} \in H^{1}(V / \Sigma, \overline{\operatorname{Br}}(\psi))^{+}$ and $\widehat{P}^{t}\left[\sigma_{1}\right]^{*}=\left[e_{1}\right]^{*} \in \mathrm{DG}(\psi)^{+}, \widehat{P}^{t}$ preserves order.

Finally,

$$
\begin{aligned}
\widehat{F}_{L_{\psi}}^{*}: \lim _{\longrightarrow}\left(F_{M_{\psi}}^{*}: H^{1}\left(\mathbb{T}^{d}, \operatorname{Br}(\psi)\right) \emptyset\right) & =\operatorname{PDG}(\psi) \\
& \rightarrow \underline{\lim }\left(F_{\left.A\right|_{V}}^{*}: H^{1}(V / \Sigma, \overline{\operatorname{Br}}(\psi)) \emptyset\right)
\end{aligned}
$$




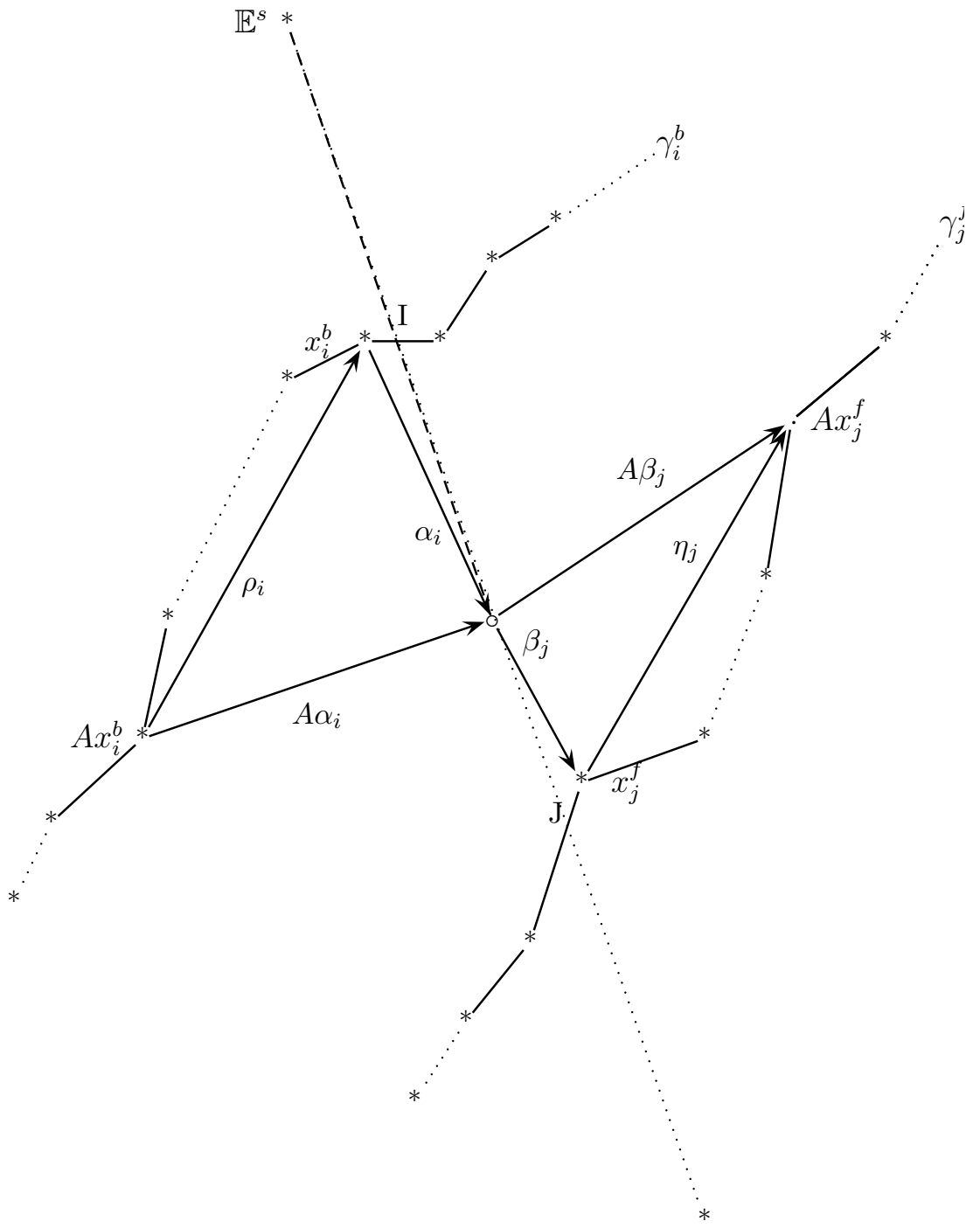

Fig. 1. A homology

defined by $\widehat{F}_{L_{\psi}}^{*}([(k, g)])=\left[\left(k, F_{L_{\psi}}^{*}(g)\right)\right]$ is an ordered isomorphism (that commutes with the shifts), which embeds $\left(\operatorname{PDG}(\psi), \operatorname{PDG}(\psi)^{+}\right)$into $(\mathrm{DG}(\psi)$, $\left.\operatorname{DG}(\psi)^{+}\right)$.

\section{References}

[AP] J. E. Anderson and I. F. Putnam, Topological invariants for substitution tilings and their associated $C^{*}$-algebras, Ergodic Theory Dynam. Systems 18 (1998), 509-537. 
[Aus] J. Auslander, Minimal Flows and Their Extensions, North-Holland Math. Stud. 153, North-Holland, Amsterdam, 1988.

[BD1] M. Barge and B. Diamond, A complete invariant for the topology of one-dimensional substitutive tiling spaces, Ergodic Theory Dynam. Systems 21 (2001), $1333-1358$.

[BD2] -, 一, Proximality in Pisot tiling spaces, Fund. Math. 194 (2007), 191-238.

[BBK] V. Baker, M. Barge, and J. Kwapisz, Geometric realization and coincidence for reducible nonunimodular Pisot tiling spaces with an application to $\beta$-shifts, Ann. Inst. Fourier (Grenoble) 56 (2006), 2213-2248.

[BDH] M. Barge, B. Diamond, and C. Holton, Asymptotic orbits of primitive substitutions, Theoret. Comput. Sci. 301 (2003), 439-450.

[BK] M. Barge and J. Kwapisz, Geometric theory of unimodular Pisot substitutions, Amer. J. Math. 128 (2006), 1219-1282.

[BSm] M. Barge and M. Smith, Augmented dimension groups and ordered cohomology, Ergodic Theory Dynam. Systems 29 (2009), 1-35.

[BSw1] M. Barge and R. Swanson, New techniques for classifying Williams solenoids, Tokyo J. Math. 30 (2007), 139-157 .

[BSw2] - - , Rigidity in one dimensional tiling spaces, Topology Appl. 154 (2007), 3095-3099.

[BT] E. Bombieri and J. E. Taylor, Which distributions of matter diffract?, J. Physiques 47 (1986), Suppl. Colloq. C3, 19-28.

[CE1] T. Carlsen and S. Eilers, Matsumoto K-groups associated with certain shift spaces, Documenta Math. 9 (2004), 639-671.

[CE2] - - , Augmenting dimension group invariants for substitutional dynamical systems, Ergodic Theory Dynam. Systems 24 (2004), 1015-1039.

[D] F. M. Dekking, The spectrum of dynamical systems arising from substitutions of constant length, Z. Wahrsch. Verw. Gebiete 41 (1977/78), 221-239.

[E1] S. Eilers, www.math.ku.dk/ eilers/myprograms.html.

[E2] - $C^{*}$-algebras associated to dynamical systems, Discrete Contin. Dynam. Systems 15 (2006), 177-192.

[ERR] S. Eilers, G. Restorff and E. Ruiz, Classification of extensions of classifiable $C^{*}$-algebras, preprint, 2007.

[LMS] J.-Y. Lee, R. Moody and B. Solomyak, Pure point dynamical and diffraction spectra, Ann. H. Poincaré 3 (2002), 1003-1018.

[M] K. Matsumoto, K-theory associated with subshifts, Math. Scand. 82 (1998), 237255 .

[Wi] R. F. Williams, Classification of 1-dimensional attractors, in: Proc. Sympos. Pure Math. 14, Amer. Math. Soc., 1970, 341-361.

Department of Mathematics

Montana State University

Bozeman, MT 59717, U.S.A.

E-mail: barge@math.montana.edu

swanson@math.montana.edu
Department of Mathematics College of Charleston Charleston, SC 29424, U.S.A. E-mail: diamondb@cofc.edu

Received 13 June 2008;

in revised form 3 June 2009 